\title{
On the Nonlinear Behavior of the Piezoelectric Coupling on Vibration-Based Energy Harvesters
}

\author{
Luciana L. Silva, ${ }^{1}$ Marcelo A. Savi, ${ }^{2}$ Paulo C. C. Monteiro Jr., ${ }^{3}$ and Theodoro A. Netto ${ }^{3}$ \\ ${ }^{1}$ Department of Mechanical Engineering, CEFET-RJ, 20.271.110 Rio de Janeiro, RJ, Brazil \\ ${ }^{2}$ Department of Mechanical Engineering, COPPE, Universidade Federal do Rio de Janeiro, 21.941.972 Rio de Janeiro, RJ, Brazil \\ ${ }^{3}$ Department of Ocean Engineering, COPPE, Universidade Federal do Rio de Janeiro, 21.945.970 Rio de Janeiro, RJ, Brazil
}

Correspondence should be addressed to Paulo C. C. Monteiro Jr.; camara@lts.coppe.ufrj.br

Received 1 April 2015; Revised 12 July 2015; Accepted 30 July 2015

Academic Editor: Federica Tubino

Copyright (C) 2015 Luciana L. Silva et al. This is an open access article distributed under the Creative Commons Attribution License, which permits unrestricted use, distribution, and reproduction in any medium, provided the original work is properly cited.

\begin{abstract}
Vibration-based energy harvesting with piezoelectric elements has an increasing importance nowadays being related to numerous potential applications. A wide range of nonlinear effects is observed in energy harvesting devices and the analysis of the power generated suggests that they have considerable influence on the results. Linear constitutive models for piezoelectric materials can provide inconsistencies on the prediction of the power output of the energy harvester, mainly close to resonant conditions. This paper investigates the effect of the nonlinear behavior of the piezoelectric coupling. A one-degree of freedom mechanical system is coupled to an electrical circuit by a piezoelectric element and different coupling models are investigated. Experimental tests available in the literature are employed as a reference establishing the best matches of the models. Subsequently, numerical simulations are carried out showing different responses of the system indicating that nonlinear piezoelectric couplings can strongly modify the system dynamics.
\end{abstract}

\section{Introduction}

Vibration-based energy harvesting is a promising area where available mechanical vibration energy is converted into electrical energy that can be employed for different purposes. Usually, piezoelectric materials are employed for electromechanical conversion [1-3]. Different kinds of structures can be considered for this aim including bridges, buildings, airplanes, and cars $[1,4,5]$.

The general idea of energy harvesting is the objective of several research efforts $[1,4,6]$. Theoretical and experimental studies investigate the design and performance optimization of vibration-based energy harvesters [7-9].

The proper description of electromechanical coupling is an essential point in the energy harvesting analysis. Experimental tests point to a nonlinear constitutive behavior. Nevertheless, it is usual to adopt a linear relation between strain and electrical field. Under this assumption, there is a single constant for all values of strain-electrical field, known as the coupling coefficient. Dutoit and Wardle [7] showed that the use of linear constitutive relations underpredicts the experimental voltage produced from energy harvesting devices. Crawley and Anderson [10] presented experimental results by considering nonlinear behavior of the strainelectrical field, providing the evidence that a linear model is not valid for large strains. Triplett and Quinn [8] treated a dynamical system with nonlinear stiffness and the piezoelectric coupling coefficient is described by a linear dependence on the induced strain. The analysis of the power generated by the harvesting system suggests that nonlinear effects have considerable influence on the results [7]. Silva et al. [11] investigated the effect of hysteretic behavior of piezoelectric coupling, comparing results with linear and nonlinear models, suggesting that there is an optimum hysteretic behavior that increases the power output of the energy harvesters.

Considerable efforts have been made to improve the power harvesting system using nonlinear approach of electrical extraction circuits $[12,13]$. Nonlinear switching harvesting techniques have been developed, such as the parallel SSHI (synchronized switching harvesting on an inductor) and 


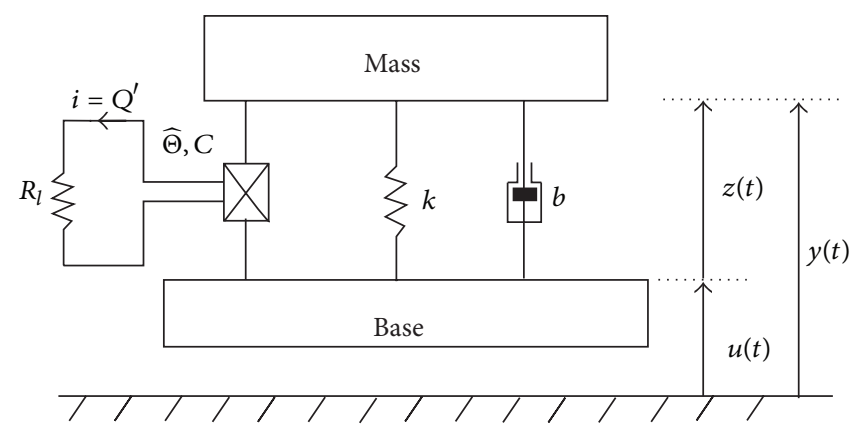

FIGURE 1: Archetypal model of the vibration-based energy harvesting system [8].

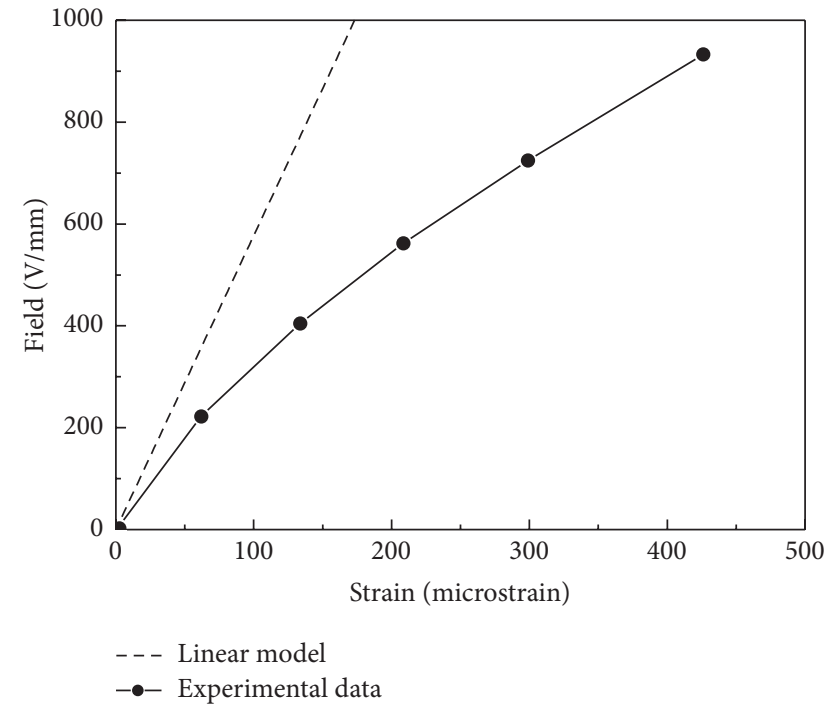

(a)

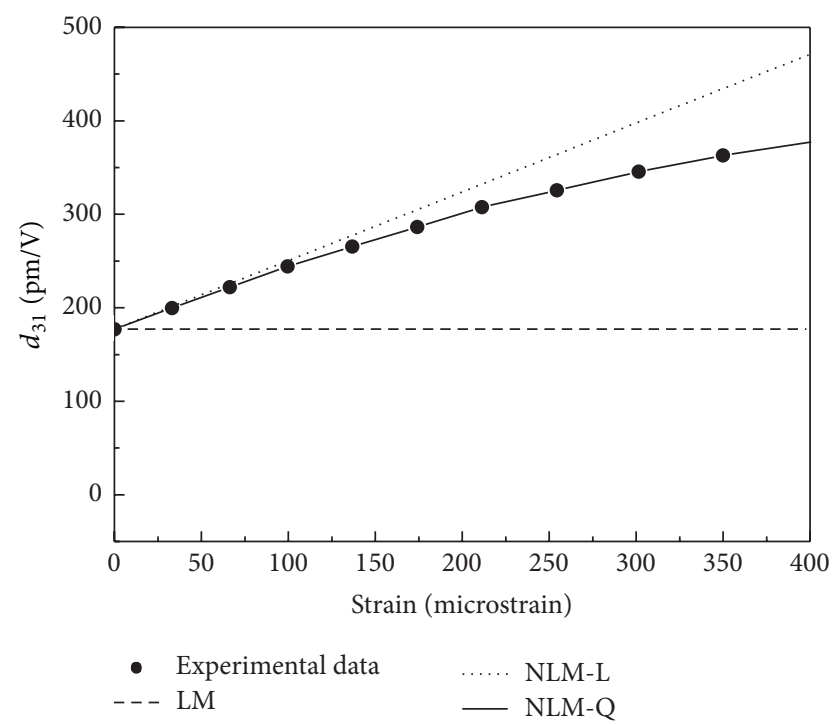

(b)

FIGURE 2: Electromechanical behavior of piezoelectric materials [10]. Electric field-strain curve (a) and $d_{31}$-strain curve (b).

series SSHI [14]. Lefeuvre et al. [15] showed that the Synchronous Electric Charge Extraction (SECE) could enhance the electromechanical conversion when compared with the classical extraction technique $[16,17]$.

Stanton et al. [18] proposed a model for the nonlinear piezoelectric response of an electroelastic energy harvester using a quadratic dependence of piezoelectric coupling coefficient on the induced strain. Experimental tests are performed showing a good agreement between numerical and experimental data.

In general, it is possible to say that nonlinear effects are being considered in order to enhance power harvested. It is usual to introduce a nonlinear compliance to extend the coupling between the environmental excitation and the harvester to a wider range of frequencies [19-21]. In this regard, it is also important to have a proper comprehension of the energy harvesting system dynamics. Random excitations are also important in order to establish a proper comprehension and applicability of energy harvesting system, being the objective of some references $[3,22]$.
This paper deals with a comparison among the effect of piezoelectric coupling nonlinearities in energy harvesting systems. Distinct models are employed and results are compared with experimental data available in literature. An archetypal model composed of a one-degree of freedom mechanical system connected to an electrical circuit by a piezoelectric element is adopted. Numerical results obtained from linear and nonlinear models are compared with experimental data due to Kim et al. [23]. Results show that nonlinear description presents better matches with experimental data, reducing inconsistencies predicted by linear models especially near resonance conditions. Afterward, dynamics of the nonlinear system is investigated showing typical characteristics that need to be properly understood. Dynamical jumps represent a critical situation where nonlinear resonant response can be associated with abrupt changes.

\section{Vibration-Based Energy Harvesting}

An archetypal model to describe the vibration-based energy harvesting system consists of a mechanical system connected 


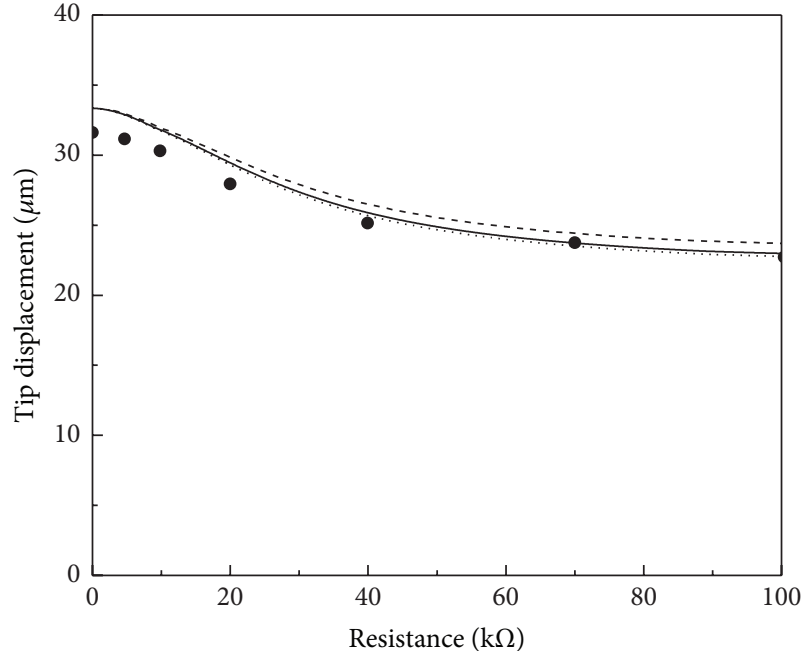

- Experimental --- NLM-L

.... LM NLM-Q

(a)

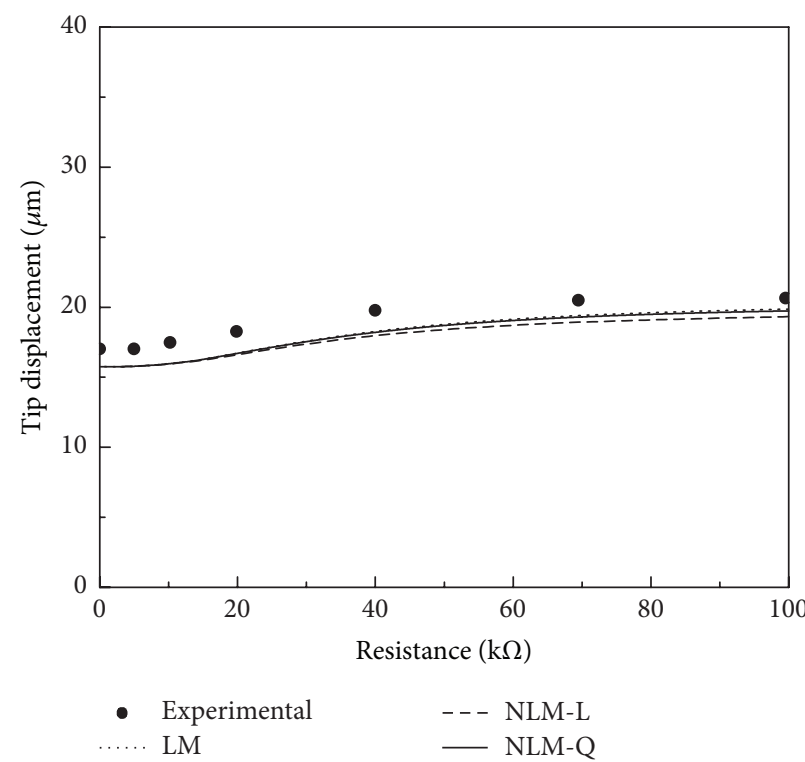

(c)

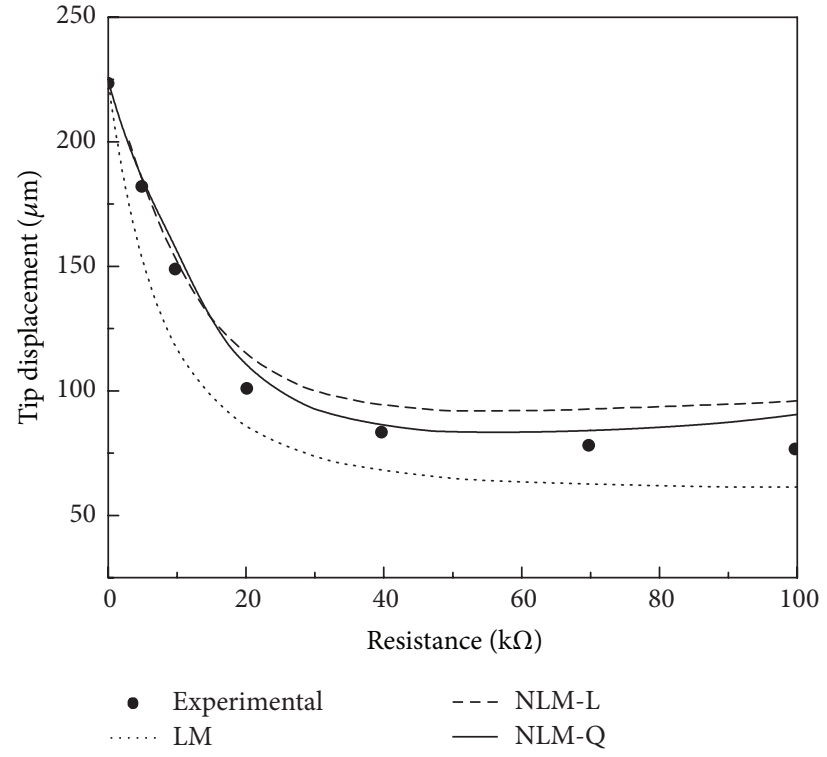

(b)

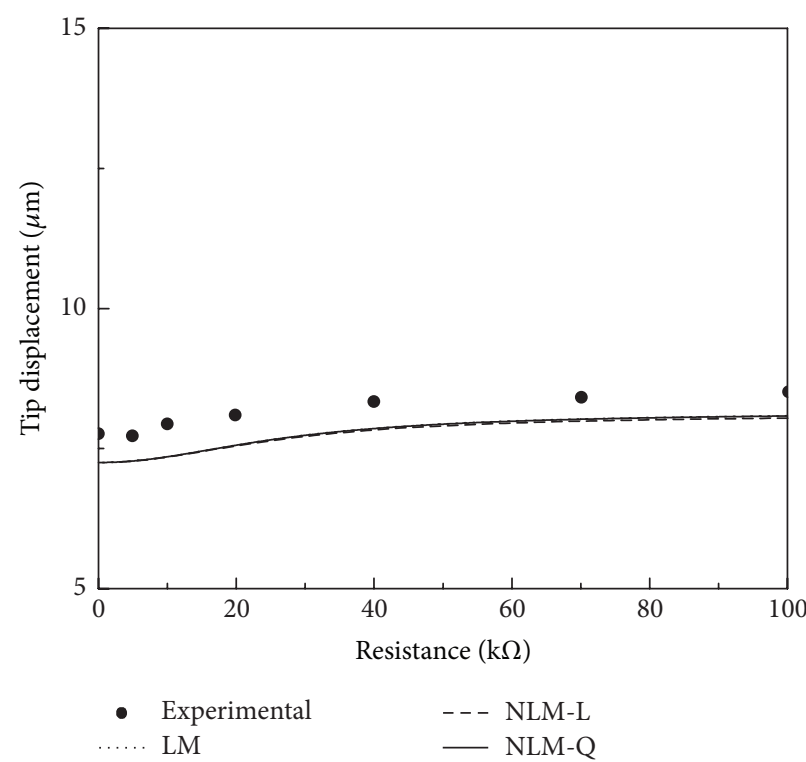

(d)

FiguRE 3: Results of tip displacement versus the electrical resistive load at different frequencies (a) $95 \mathrm{~Hz}$, (b) $109.5 \mathrm{~Hz}$ (resonance frequency), (c) $135 \mathrm{~Hz}$, and (d) $160 \mathrm{~Hz}$. Experimental data (scatter), LM (scatter line), NLM-L (dashed line), and NLM-Q (solid line) models.

to an electrical circuit by a piezoelectric element, responsible for the electromechanical conversion (Figure 1). A massspring-damper oscillator with mass, $m$, stiffness $k$, and a linear viscous coefficient $b$ represents the mechanical system. This system is subjected to a base excitation $u=u(t)$, and the mass displacement is represented by $y ; z$ is the mass displacement relative to the base. An electrical resistance, $R_{l}$, represents the electrical circuit and $V$ denotes the voltage across the piezoelectric element. The electromechanical coupling is provided by the piezoelectric element being represented by $\widehat{\Theta}$.
Therefore, the system dynamics may be described by the following equations of motion:

$$
\begin{aligned}
m \ddot{z}+b \dot{z}+k z-\widehat{\Theta} V & =-B_{f} \ddot{u}, \\
\widehat{\Theta} \dot{z}+C \dot{V}+\frac{1}{R_{l}} V & =0,
\end{aligned}
$$

where $(\dot{\boldsymbol{\square}}) \equiv d(\boldsymbol{\square}) / d t, B_{f}$ is the forcing function related to base excitation being defined to represent the inertial loading of the device due to base excitation [23]. The electromechanical coupling provided by the piezoelectric element, $\widehat{\Theta}$, needs 


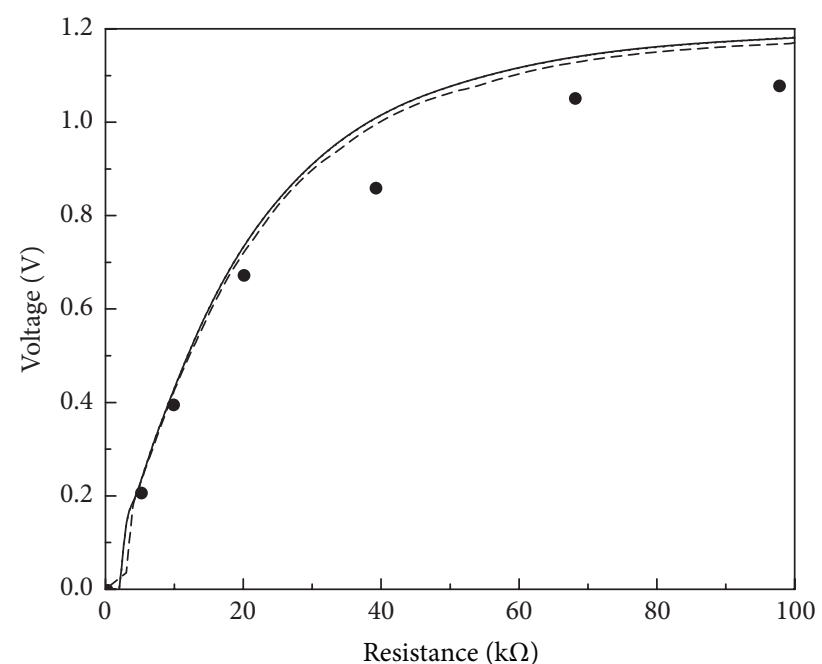

- Experimental --- NLM-L LM

(a)

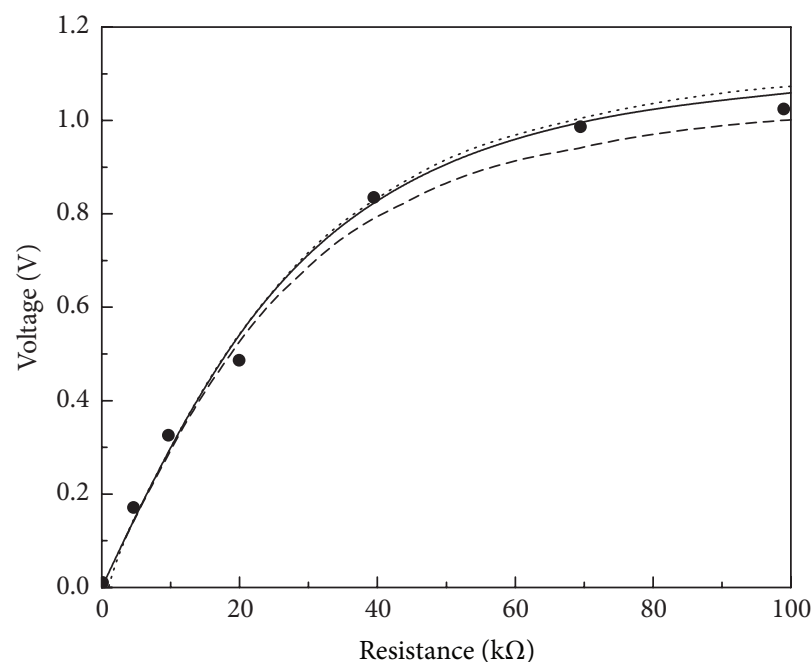

- Experimental - - - NLM-L - NLM-Q

(c)

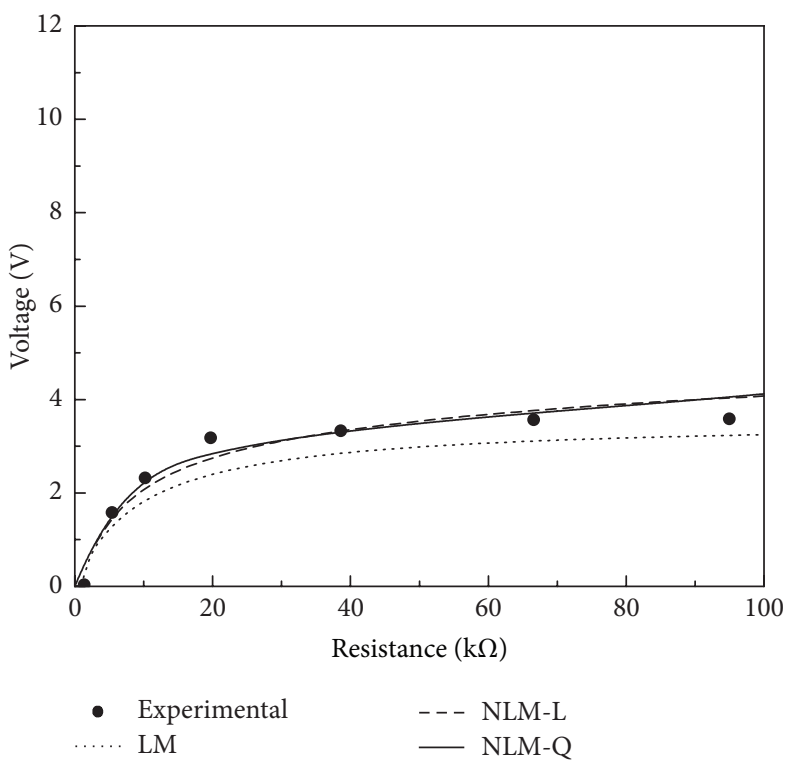

(b)

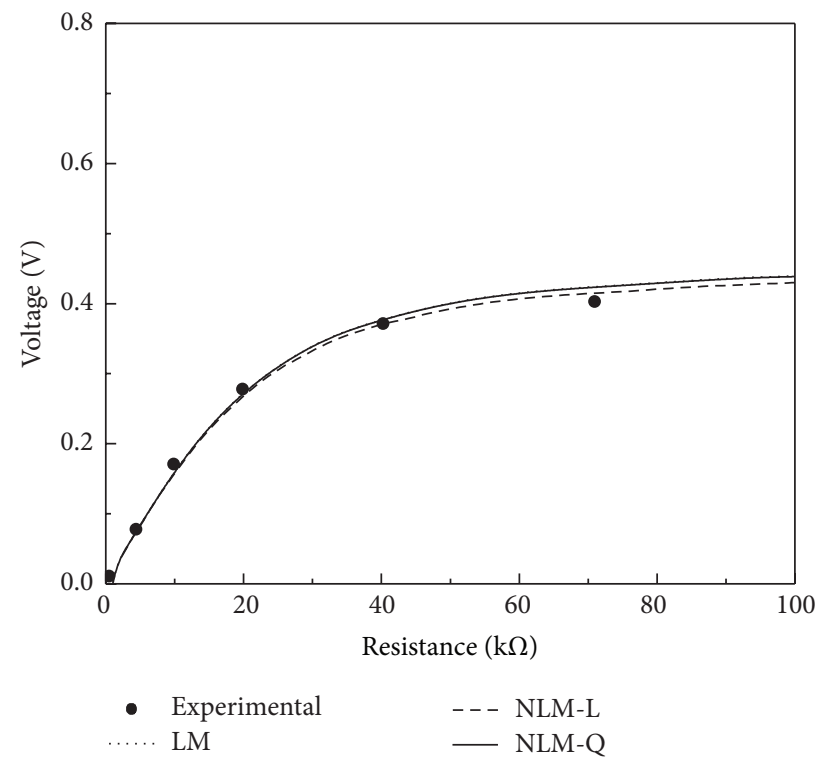

(d)

FIGURE 4: Results of voltage versus the electrical resistive load at different (a) $95 \mathrm{~Hz}$, (b) $109.5 \mathrm{~Hz}$ (resonance frequency), (c) $135 \mathrm{~Hz}$, and (d) $160 \mathrm{~Hz}$. Experimental data (scatter), LM (scatter line), NLM-L (dashed line), and NLM-Q (solid line) models.

to be properly described by some constitutive equation. The next section treats this modeling.

2.1. Piezoelectric Constitutive Equations. The general description of the $3 \mathrm{D}$ behavior of piezoelectric materials can be expressed by considering the strain, $S_{i}$, the stress, $T_{i}$, the electric displacement, $D_{i}$, and the applied field, $E_{i}$. The elastic compliance, piezoelectric coupling, and permittivity matrices are denoted, respectively, by $s_{i j}, d_{i j}$, and $\varepsilon_{i j}$. The superscript " $E$ " stands for measurements at zero or constant electric field and " $T$ " denotes measurements that are taken at null or constant stress. Therefore, the 3D constitutive equations for inverse and direct effects are given by

$$
\begin{aligned}
& S_{i}=s_{i j}^{E} T_{j}+d_{m i} E_{m} \text { (inverse effect), } \\
& D_{m}=d_{m i} T_{i}+\varepsilon_{m k}^{T} E_{k} \text { (direct effect). }
\end{aligned}
$$

Coefficient $d_{i j}$ establishes the relation between electric and mechanical fields. Experimental data shows that this electromechanical behavior is strongly dependent on the electric 


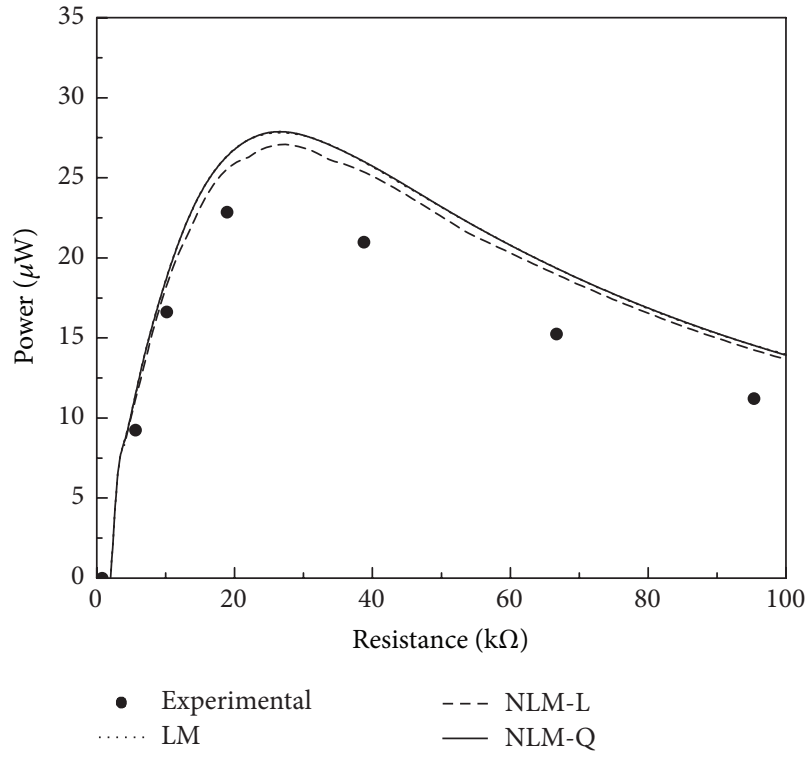

(a)

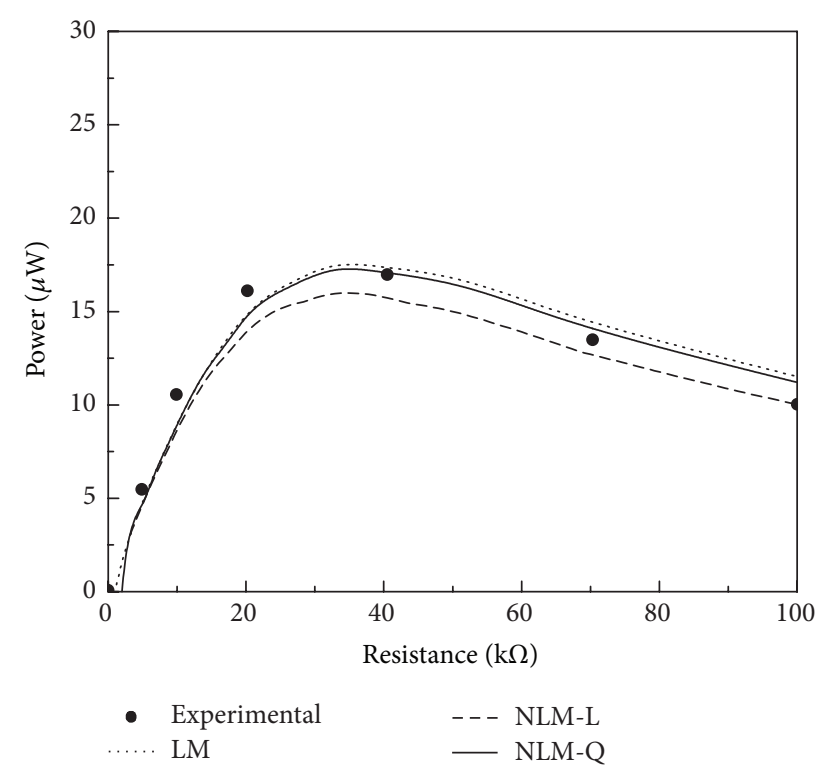

(c)

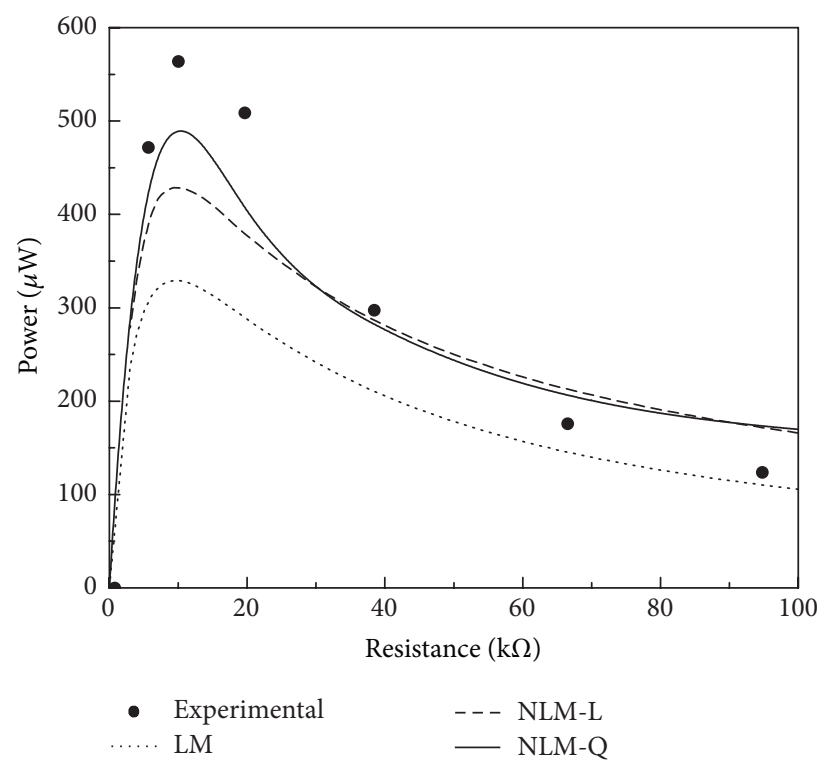

(b)

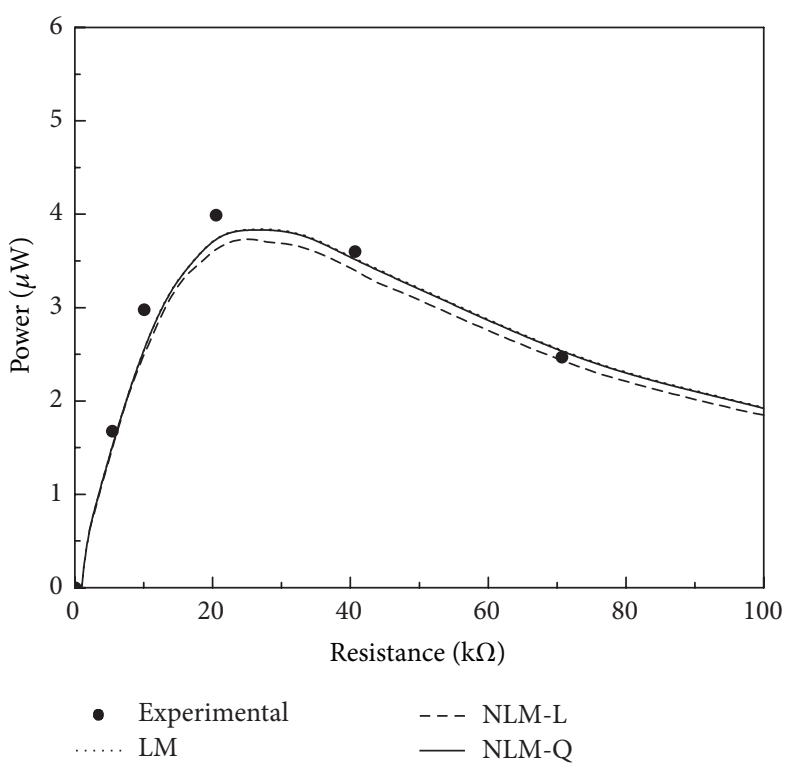

(d)

FIGURE 5: Results of power harvested versus the electrical resistive load at different frequencies (a) $95 \mathrm{~Hz}$, (b) $109.5 \mathrm{~Hz}$ (resonance frequency), (c) $135 \mathrm{~Hz}$, and (d) $160 \mathrm{~Hz}$. Experimental data (scatter), LM (scatter line), NLM-L (dashed line), and NLM-Q (solid line) models.

field intensity, as shown in Figure 2(a) from experimental data of Crawley and Anderson [10]. Different models can be adopted to match experimental data. A linear constitutive relation can be represented by a single constant value for all strain-electric fields as indicated in Figure 2(b) (LM). Distinct nonlinear descriptions can be employed to represent this constitutive response but two possibilities seem to be direct: the first one assumes that the piezoelectric coupling coefficient has a linear dependence of the induced strain (NLM-L), as proposed by Triplett and Quinn [8] (dotted curve in Figure 2(b)). An alternative nonlinear description is a quadratic approximation of the induced strain (NLMQ) [18]. This paper considers these three possibilities for the constitutive description of the piezoelectric behavior using a general equation that follows

$$
\widehat{\Theta}(z)=\eta\left(1+\alpha_{1}|z|+\alpha_{2} z^{2}\right),
$$

where $\eta$ and $\alpha_{i}(i=1,2)$ are, respectively, linear and nonlinear piezoelectric coupling coefficients. Note that the definition 


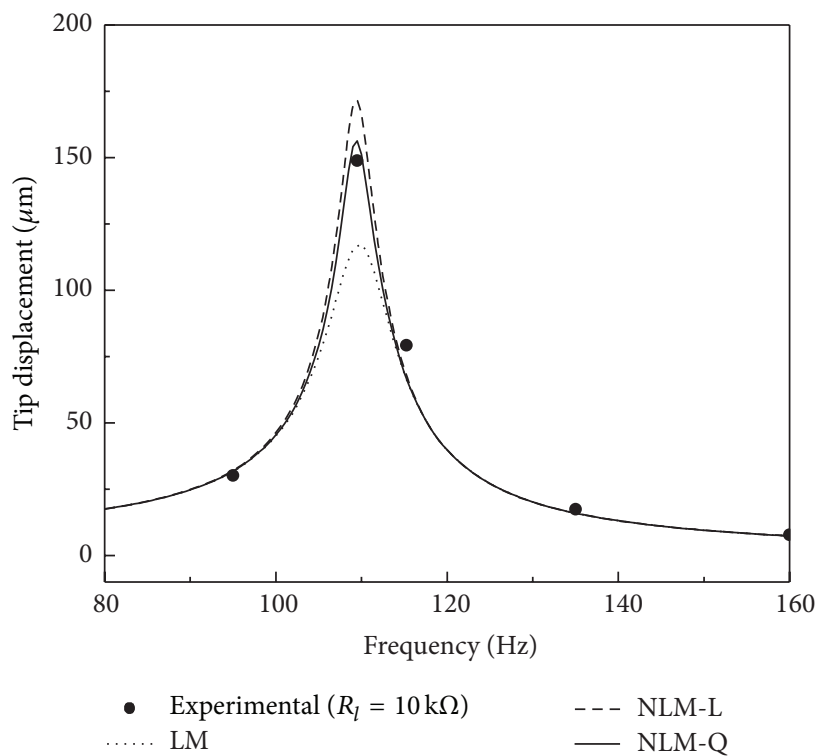

(a)

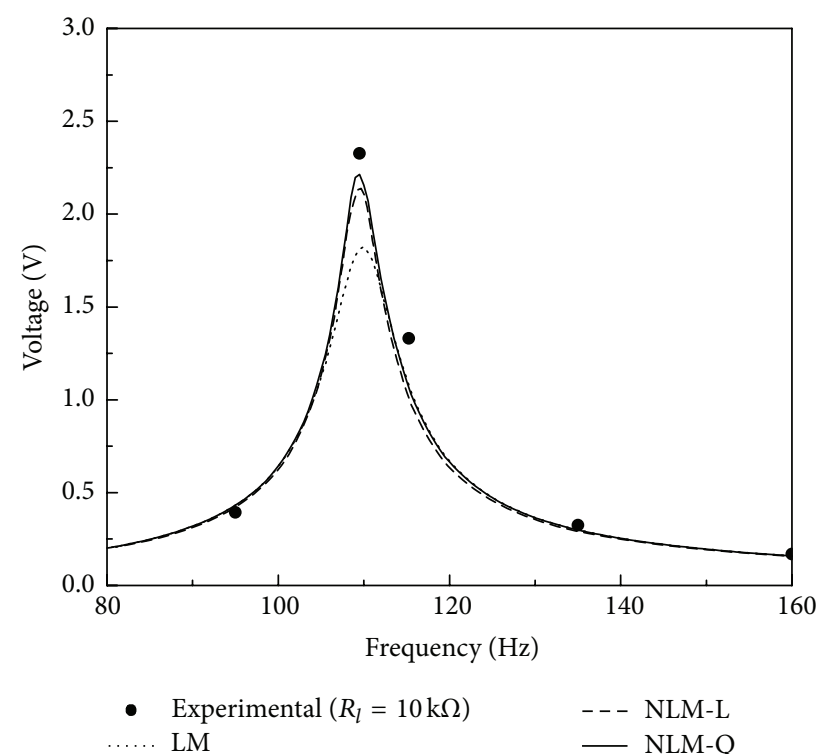

(b)

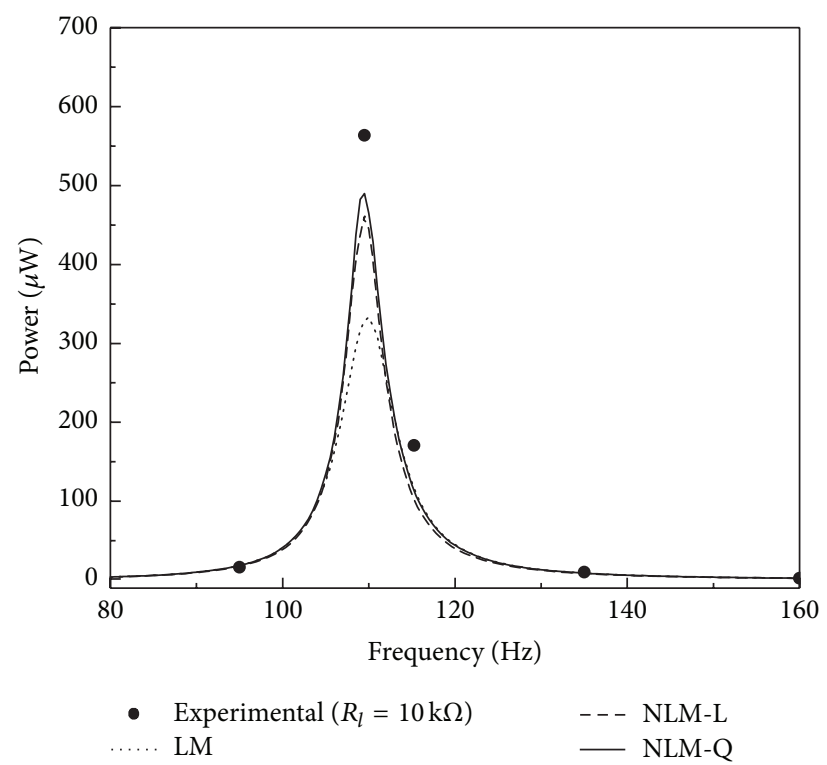

(c)

Figure 6: Results of experimental data (scatter), LM (scatter line), NLM-L (dashed line), and NLM-Q (solid line) models of (a) tip displacement, (b) voltage, and (c) power versus frequency at electrical resistive load of $10 \mathrm{k} \Omega$.

of the linear model (LM) assumes that $\alpha_{1}$ and $\alpha_{2}$ vanish. If only $\alpha_{2}$ vanishes, the equation represents a nonlinear model with a coefficient with linear dependence (NLM-L). Finally, considering that $\alpha_{1}$ and $\alpha_{2}$ do not vanish, the equation represents a nonlinear description with a coefficient with quadratic dependence (NLM-Q). Based on this constitutive model, the energy harvesting archetypal system is described by the following equations of motion:

$$
\begin{gathered}
m \ddot{z}+b \dot{z}+k z-\eta\left(1+\alpha_{1}|z|+\alpha_{2} z^{2}\right) V=-B_{f} \ddot{u}, \\
\eta\left(1+\alpha_{1}|z|+\alpha_{2} z^{2}\right) \dot{z}+C \dot{V}+\frac{1}{R_{l}} V=0 .
\end{gathered}
$$

\section{Experimental Verification}

Numerical simulations are now performed establishing a comparison among experimental data obtained from Kim et al. [23] and numerical simulations obtained from three different models: LM, NLM-L, and NLM-Q. The experimental data is obtained using a brass reinforced bending actuator, consisting of a brass substrate beam between two piezoelectric layers (PZT-5A) from Piezo Systems Inc. (model T226-A4-503X), where the resonant frequency is $109.5 \mathrm{~Hz}$ without a proof mass. All simulations are performed with the following parameters [23]: $M=0.00878 \mathrm{~kg}, B_{f}=0.006872 \mathrm{~kg}$, 


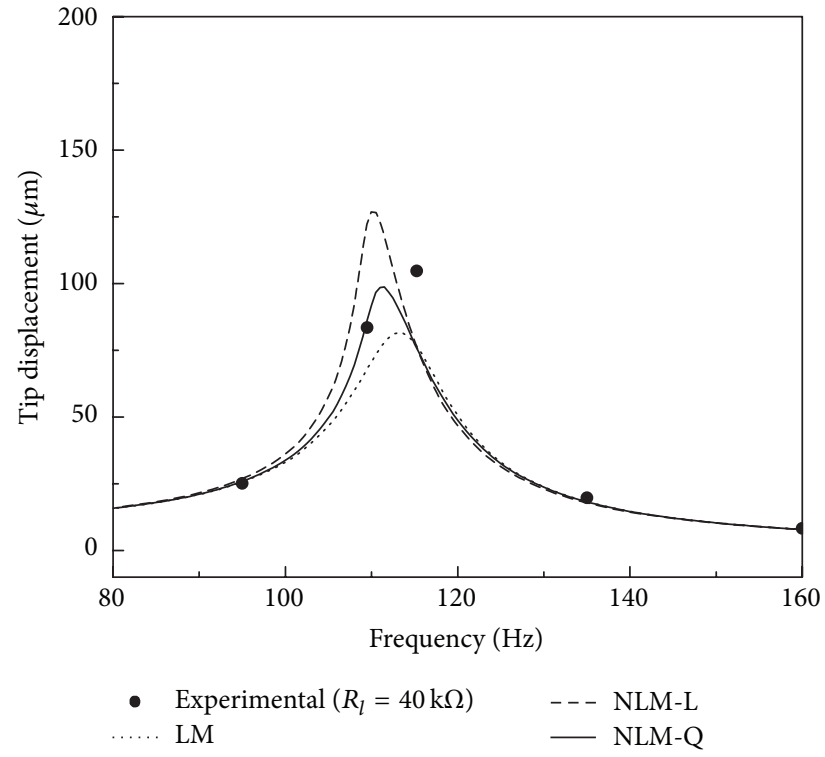

(a)

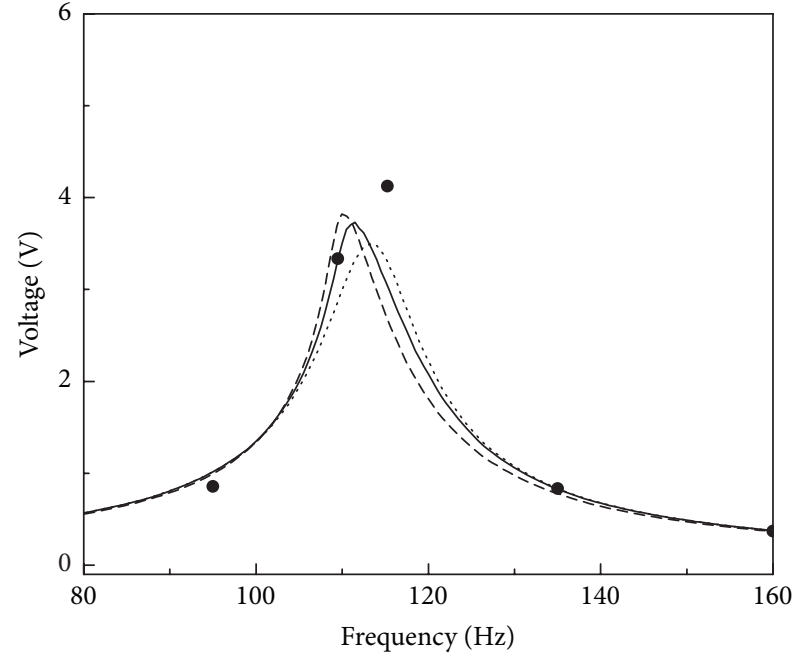

- Experimental $\left(R_{l}=40 \mathrm{k} \Omega\right) \quad--$ NLM-L LM

(b)

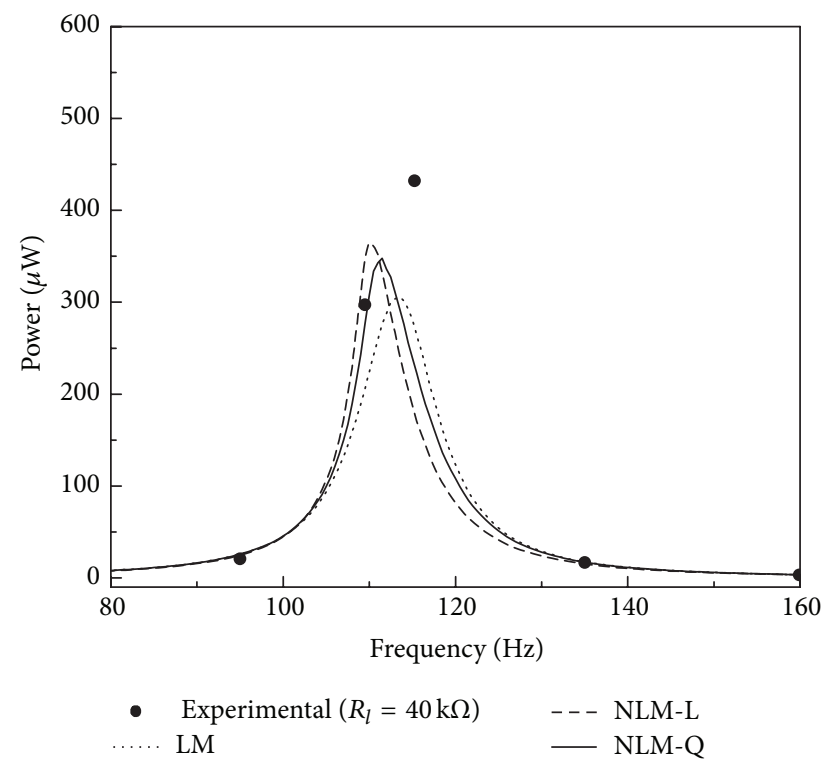

(c)

Figure 7: Results of experimental data (scatter), LM (scatter line), NLM-L (dashed line), and NLM-Q (solid line) models of (a) tip displacement, (b) voltage, and (c) power versus frequency at electrical resistive load of $40 \mathrm{k} \Omega$.

$b=25.0 \mathrm{Nsm}^{-1}, K=4150 \mathrm{Nm}^{-1}, \widehat{\Theta}=\eta=-0.004688 \mathrm{NV}^{-1}$, $C=4.194 \times 10^{-8} \mathrm{~F}$, and the base acceleration of $2.5 \mathrm{~ms}^{-2}$.

A nonlinear least-squares algorithm is used to find an optimal fit to the experimental data for the full range of excitation frequencies and resistances. Nonlinear models also need to define the coupling coefficients, $\alpha_{1}$ and $\alpha_{2}$. A nonlinear least-squares algorithm is employed to determine an optimal fit to the experimental data for the full range of excitation frequencies and electrical resistances. The nonlinear coefficient values $\alpha_{1}=-8.715 \times 10^{3} \mathrm{~m}^{-1}$ and $\alpha_{2}=$ $-2.36 \times 10^{8} \mathrm{~m}^{-2}$ provide the best theoretical agreement in the mechanical voltage across the resistive load and power response in comparison with experimental data.

Initially, the energy harvesting system is analyzed by considering different electrical resistive load values for a specific frequency. Different frequency excitations are of concern: (a) $95 \mathrm{~Hz}$, (b) $109.5 \mathrm{~Hz}$ (resonant frequency), (c) $135 \mathrm{~Hz}$, and (d) $160 \mathrm{~Hz}$. All cases establish a comparison of linear (LM) and both nonlinear models (NLM-L and NLM-Q) and experimental data. Figure 3 presents tip displacement versus electrical load. Figure 4 presents the voltage-electrical load curve, while Figure 5 presents the power versus the electrical 


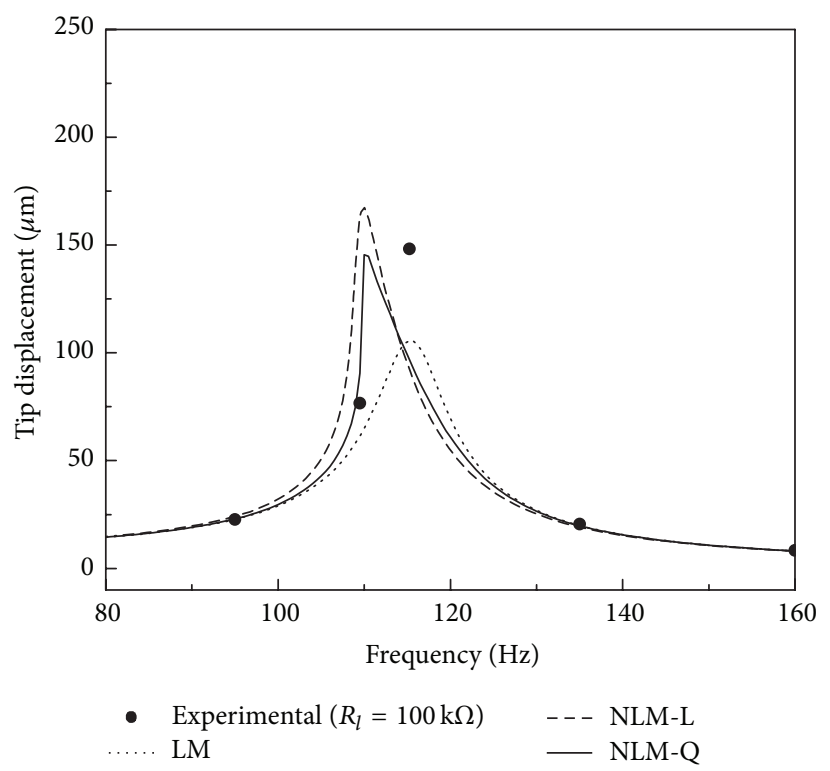

(a)

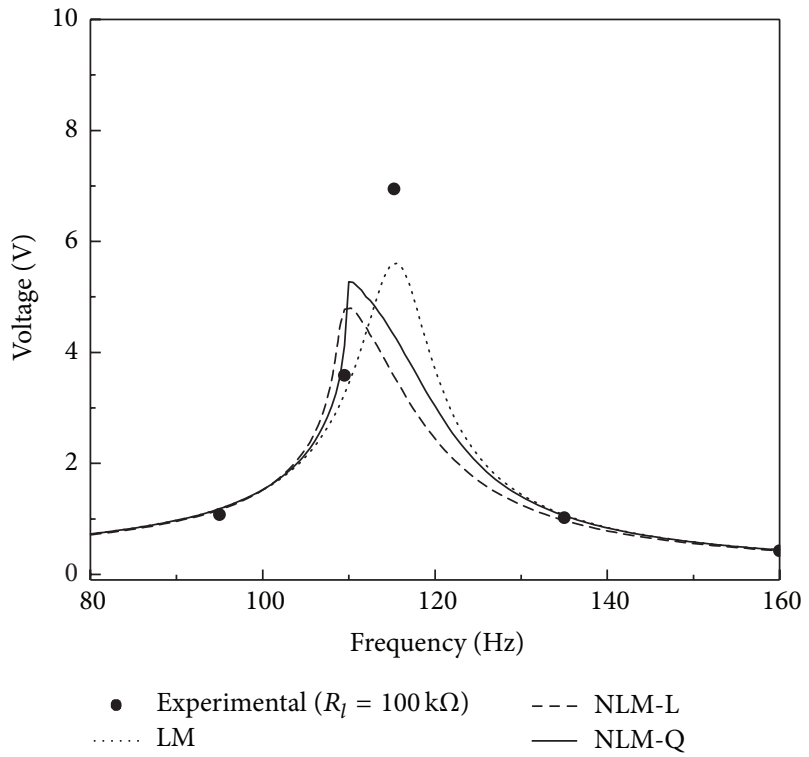

(b)

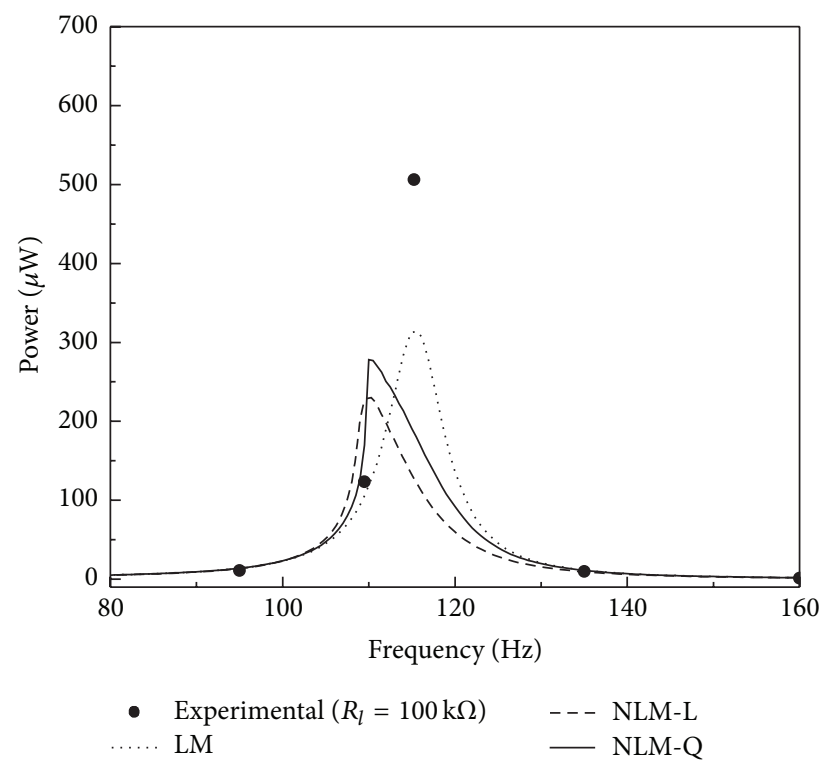

(c)

FIgURE 8: Results of experimental data (scatter), LM (scatter line), NLM-L (dashed line), and NLM-Q (solid line) models of (a) tip displacement, (b) voltage, and (c) power versus frequency at electrical resistive load of $100 \mathrm{k} \Omega$.

load. It should be observed that the linear model is in good agreement with experimental data at various electrical loadings and different operating frequencies, but as the operating frequency gets closer to resonance condition, there is a large discrepancy between the displacement, voltage, and power results obtained from linear model (LM) when compared with experimental measurements. This effect occurs since under resonant conditions, there are large induced strains, and nonlinear contribution becomes more important. In other frequencies, results of all models are quite similar due to the small values of the induced strain. A comparison between nonlinear models shows that the NLM-Q model has the better agreement with the experimental data.

This general behavior is even more evident observing power curve. Figure 5(b) shows that the peak power measured at electrical load $10 \mathrm{k} \Omega$ has the highest discrepancy compared with the LM model, $41.6 \%$. The use of the NLMQ model provides a deviation of $11.6 \%$ while NLM-L model presents $24 \%$ of difference. Since many piezoelectric harvesters are designed to operate under resonant conditions, 


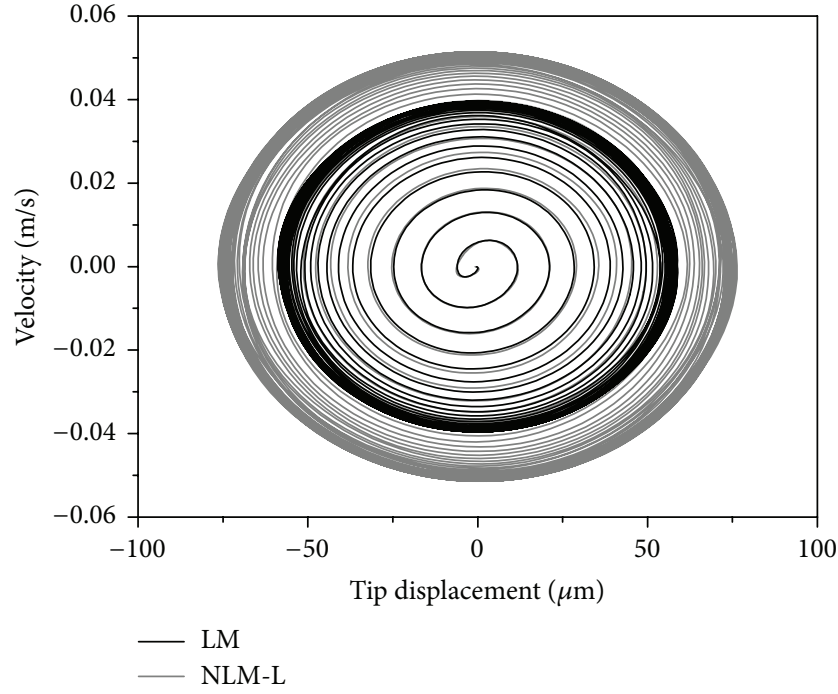

(a)

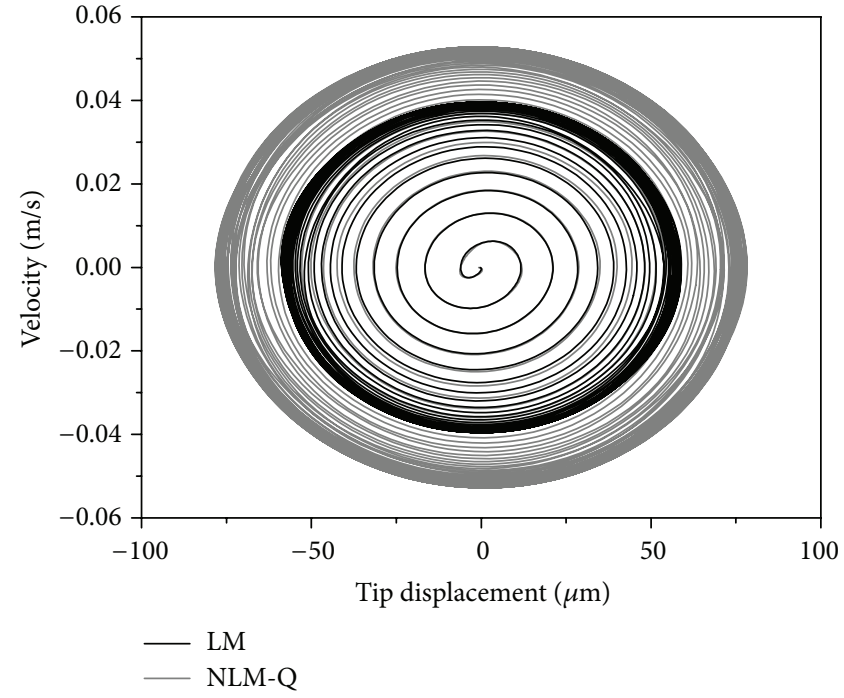

(b)

FIGURE 9: Phase space at the resonance frequency $(109.5 \mathrm{~Hz})$ and electrical load $10 \mathrm{k} \Omega$ : (a) LM and NLM-L and (b) LM and NLM-Q.

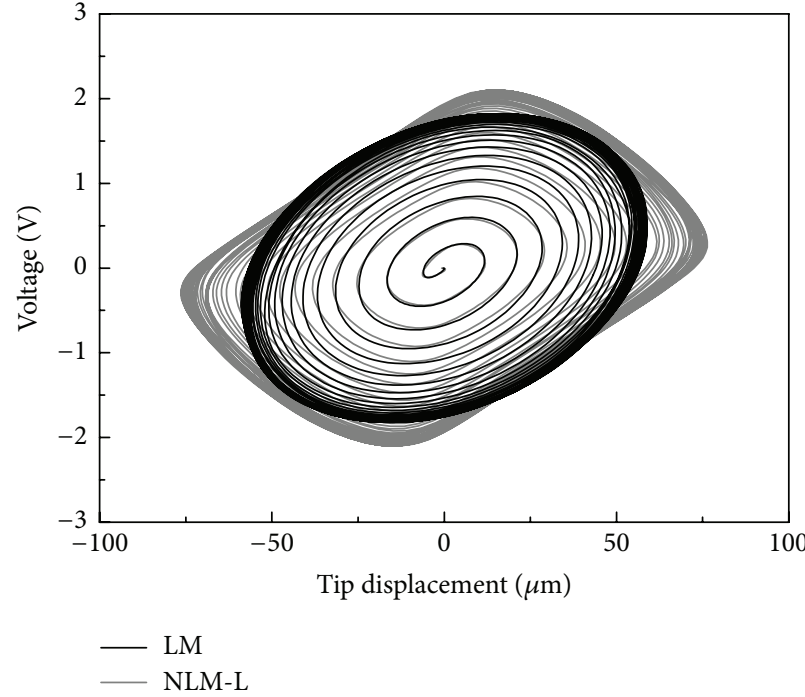

(a)

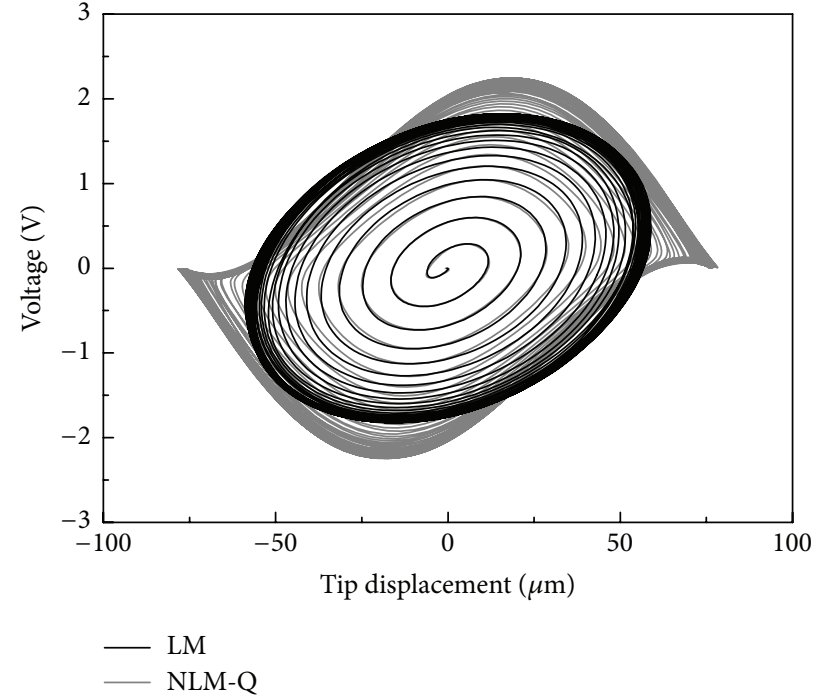

(b)

Figure 10: Voltage-displacement curve at the resonance frequency $(109.5 \mathrm{~Hz})$ and electrical load $10 \mathrm{k} \Omega$ : (a) LM and NLM-L and (b) LM and NLM-Q.

this conclusion points to the necessity of the use of a proper nonlinear model in order to obtain an appropriate power prediction.

A variation of frequency values for a specific electrical resistive load is now in focus. Figures 6-8 show results of this analysis showing, for each figure, tip displacement, voltage, and power as a function of frequency. Figure 6 considers electrical resistive load of $10 \mathrm{k} \Omega$; Figure 7 considers $40 \mathrm{k} \Omega$, while Figure 8 considers $100 \mathrm{k} \Omega$. Once again, linear and nonlinear models are compared with experimental data. When comparing results of the nonlinear models with those of the linear model, it is possible to observe that the maximum tip displacement, voltage, and power are in general greater for the nonlinear models near resonances, except when the electrical resistive load is $100 \mathrm{k} \Omega$, for voltage and power results. These results show that linear model has higher damping than the nonlinear models. When the electrical resistive load increases, even though the peak displacement is greater in the nonlinear model, the effect of the electrical resistive load becomes predominant, resulting in decreasing of voltage and power obtained from nonlinear models.

Note that the increase of the electrical resistive loads tends to shift maximum value of the tip displacement, voltage, and power curves to the left, Figures 7-8. The nonlinear curves 


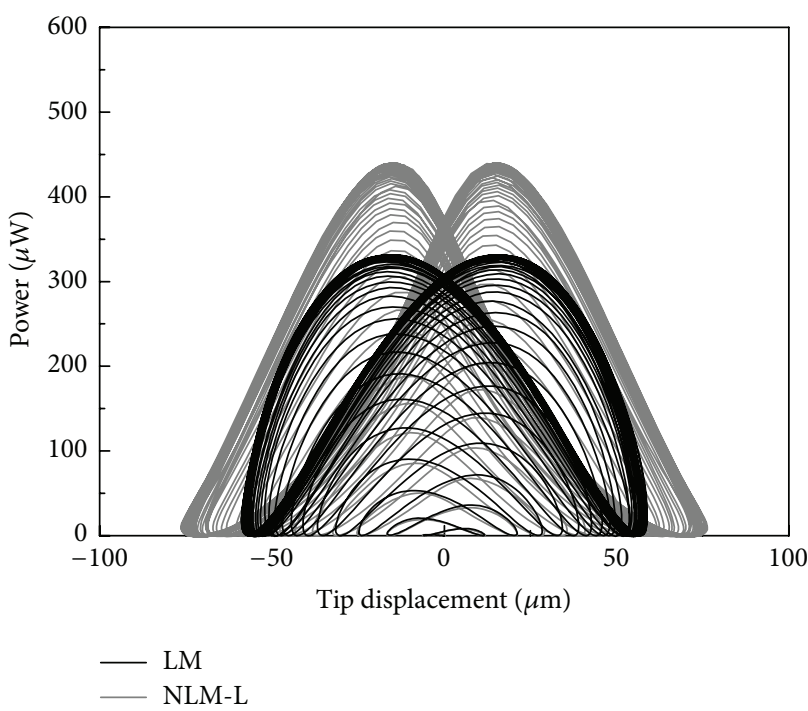

(a)

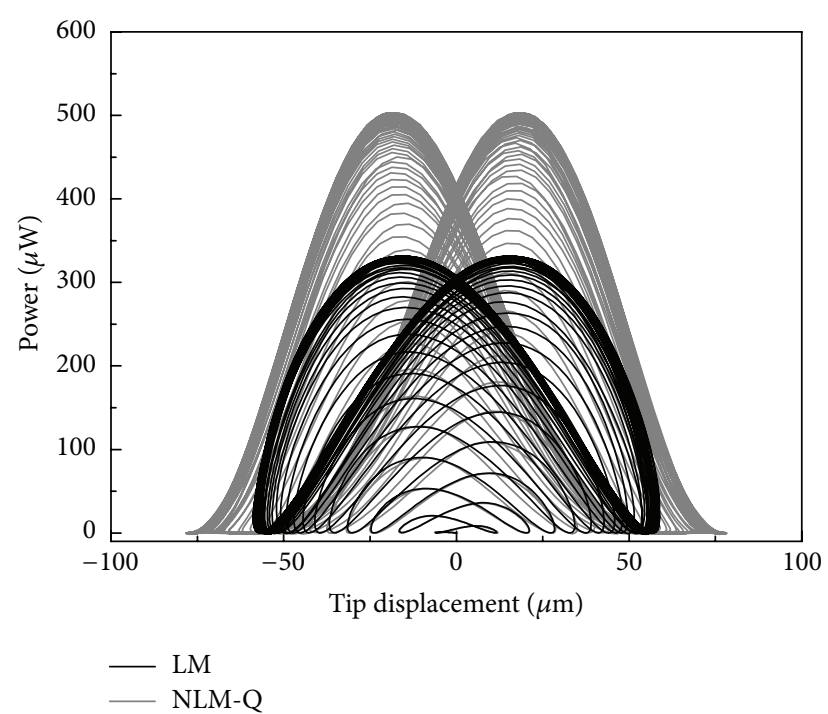

(b)

Figure 11: Power-displacement curve at the resonance frequency $(109.5 \mathrm{~Hz})$ and electrical load $10 \mathrm{k} \Omega$ comparing (a) LM and NLM-L and (b) LM and NLM-Q.

demonstrate the softening trend, resulting in the resonant behavior being pushed to lower frequencies due the elastic effects. Besides, nonlinear and linear models are not able to reproduce the value of displacement, voltage, and power for frequency $115.25 \mathrm{~Hz}$. This discrepancy increases with the increase of the electrical resistive load at the antiresonance frequency [23].

Some comparisons related to the energy harvesting system response are now investigated. Figures 9-10 show a comparison of linear and nonlinear models of the energy harvesting device at the resonance frequency $(109.5 \mathrm{~Hz})$ and electrical load $10 \mathrm{k} \Omega$. Figure 9 shows the phase space while Figure 10 shows voltage-displacement curve. This result is related to the highest discrepancy between power from experimental data and power from LM model. It is possible to observe that the vibration amplitude of the nonlinear models can be bigger than the linear model for the same amplitude force, but results between NLM-L (Figure 9(a)) and NLMQ (Figure 9(b)) are quite similar. Likewise, Figure 10 shows the voltage-displacement curve where it is possible to observe that the nonlinear models have more pronounced dissipation characteristics when compared with linear one. Figure 11 presents power response. It is noticeable that nonlinear models predict greater values when compared with linear model.

\section{A Deeper Investigation of the Dynamical Response}

The preceding section establishes a comparison among experimental data and different piezoelectric models. The main conclusion is that the nonlinear piezoelectric coupling presents a better match with experimental data. Therefore, it is important to perform a deeper investigation of the nonlinear dynamics of the energy harvesting system.

In this regard, a nondimensional version of the energy harvester mathematical model is employed. Hence, consider new coordinates $x=z / l$. Moreover, it is assumed that $V=$ $c_{v} v$, where $c_{v}$ is a constant with dimension of inverse voltage, $\omega_{0}=\sqrt{\mathrm{k} / \mathrm{m}}$ is a frequency, and the amplitude of harmonic excitation is $-B_{f} \ddot{u}=A \sin (\omega t)$. Using $\bar{k}=k / m \omega_{0}^{2}, 2 \epsilon \zeta=b /$ $m \omega_{0}, \epsilon=c_{v}^{2} C / k l^{2}, \phi=\left(l / C c_{v}\right) \widehat{\Theta}, \rho=R_{l} C \omega_{0}, \bar{\omega}=\omega / \omega_{0}, \theta=$ $\left(l / C c_{v}\right) \eta, \beta_{1}=l \alpha_{1}, \beta_{2}=l^{2} \alpha_{2}$, and $\delta=A / m l \omega_{0}^{2}$, the equations for vibration-based energy harvesting system can be rewritten as follows:

$$
\begin{aligned}
x^{\prime \prime}+2 \epsilon \zeta x^{\prime}+\bar{k} x-\epsilon \phi v & =\delta \sin (\bar{\omega} \tau), \\
\phi x^{\prime}+v^{\prime}+\frac{v}{\rho} & =0,
\end{aligned}
$$

where $\left(\boldsymbol{\square}^{\prime}\right) \equiv d(\boldsymbol{\square}) / d \tau$ and $\tau=\omega_{0} t$ is the nondimensional time and piezoelectric coupling is represented by

$$
\phi=\theta\left(1+\beta_{1}|x|+\beta_{2} x^{2}\right) .
$$

Moreover, it is important to define he instantaneous nondimensional electrical power: $P=\left(v^{\prime}\right)^{2} / \rho$.

Numerical simulations are carried out considering the following parameters: $\delta=0.1, \zeta=0.25, \epsilon=0.1$, and $\theta=$ -1.0 . Since the system dynamics has a strong dependence of nonlinear piezoelectric couplings $\left(\beta_{1}\right.$ and $\left.\beta_{2}\right)$ and the electrical resistive load, $\rho$, these parameters are varied during the analysis. Note that three different models are treated to represent the piezoelectric coupling: $\operatorname{LM}\left(\beta_{1}=\beta_{2}=0\right)$, $\operatorname{NLM}-\mathrm{L}\left(\beta_{1} \neq 0\right.$ and $\left.\beta_{2}=0\right)$, and NLM-Q $\left(\beta_{1}=0\right.$ and $\left.\beta_{2} \neq 0\right)$. Besides, nondimensional electrical resistive load 


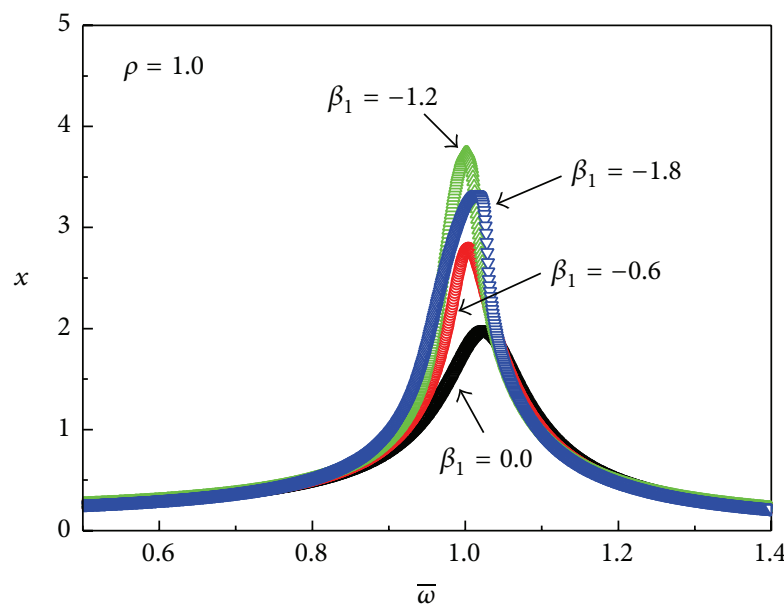

(a)

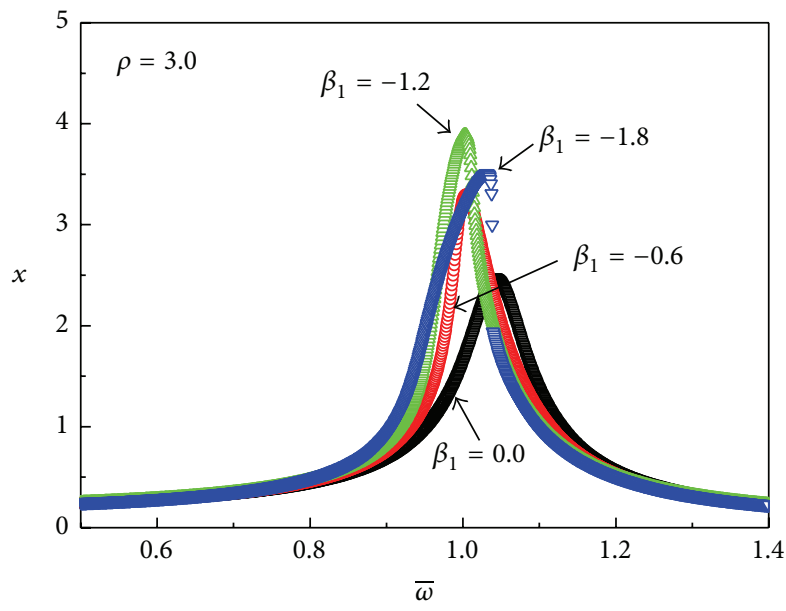

(c)

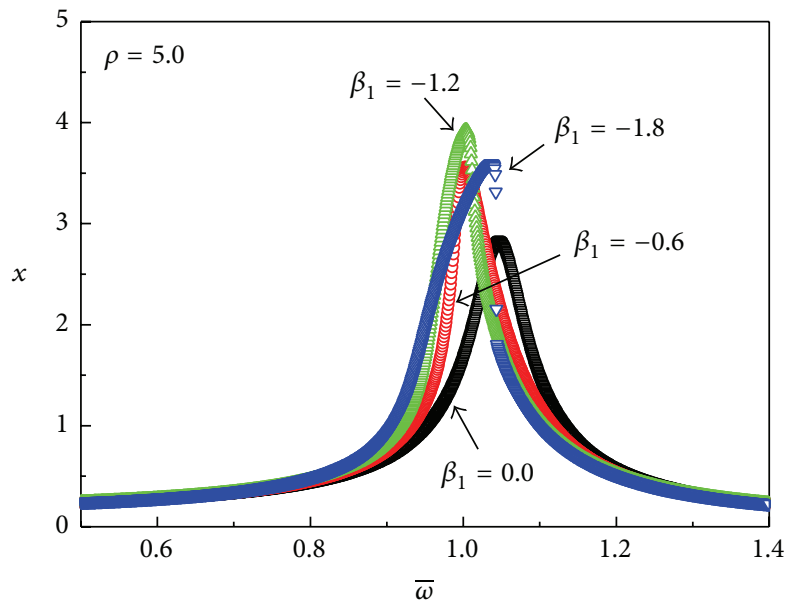

(e)

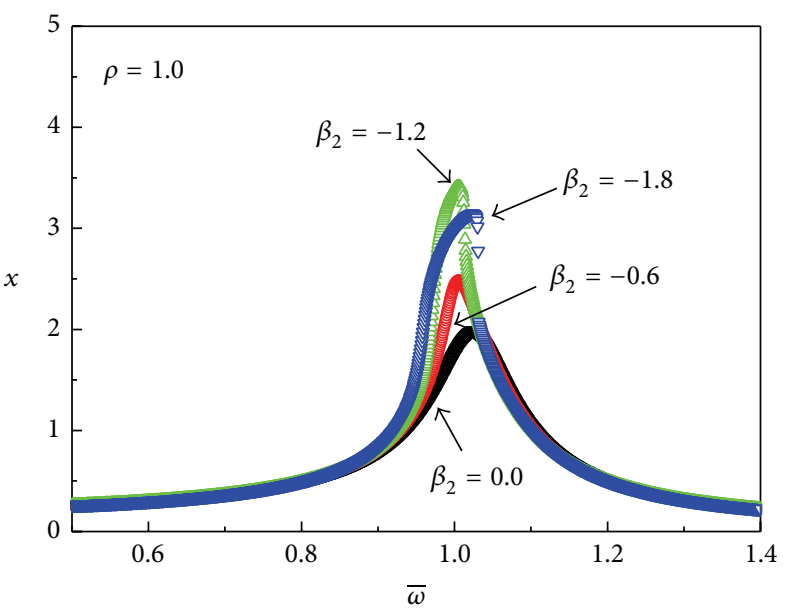

(b)

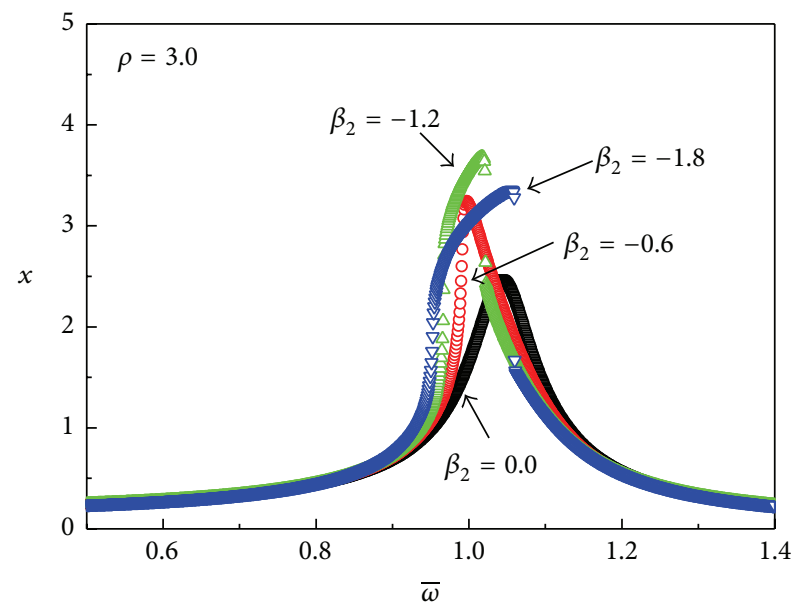

(d)

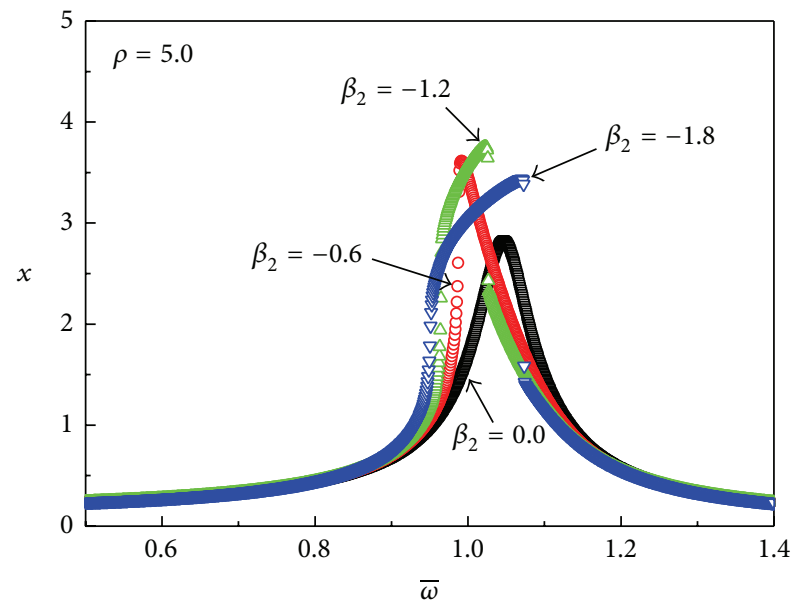

(f)

FIGURE 12: Maximum displacement-frequency curves with different nonlinear piezoelectric couplings for the LM, NLM-L, and NLM-Q models and three nondimension electrical resistive load. 


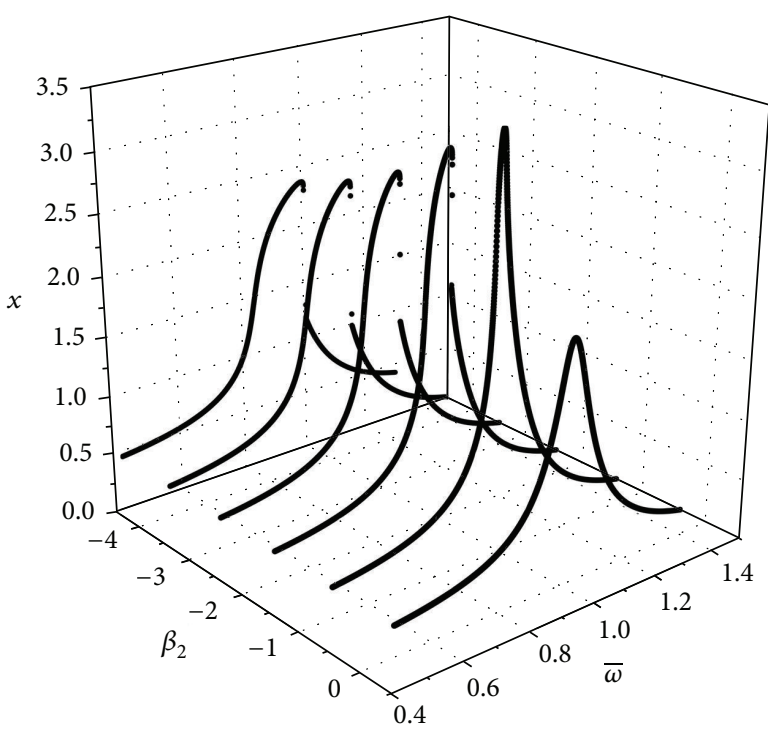

(a)

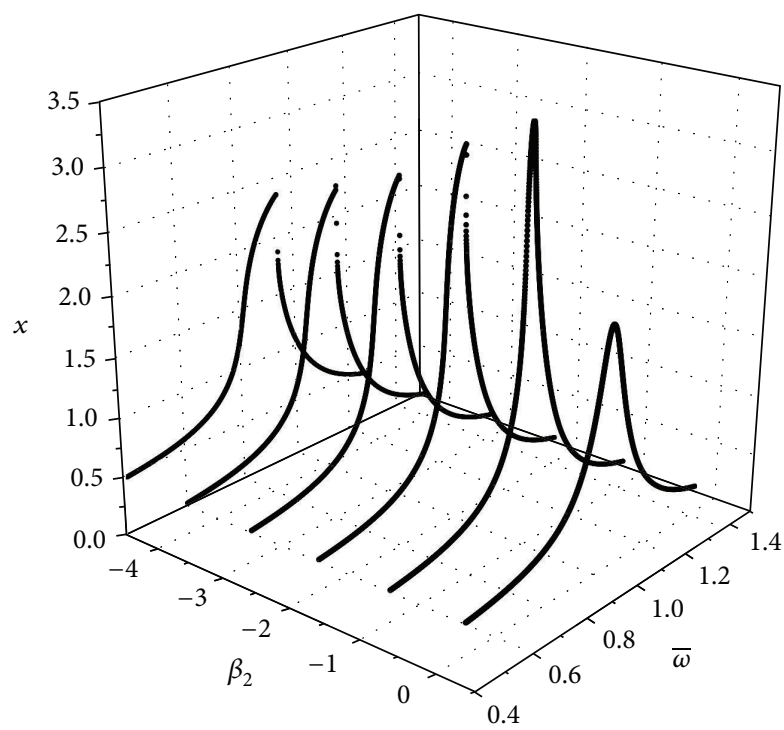

(b)

FIGURE 13: Maximum displacement-frequency curves with $\rho=1$ for the LM and NLM-Q models; (a) upsweep and (b) downsweep.

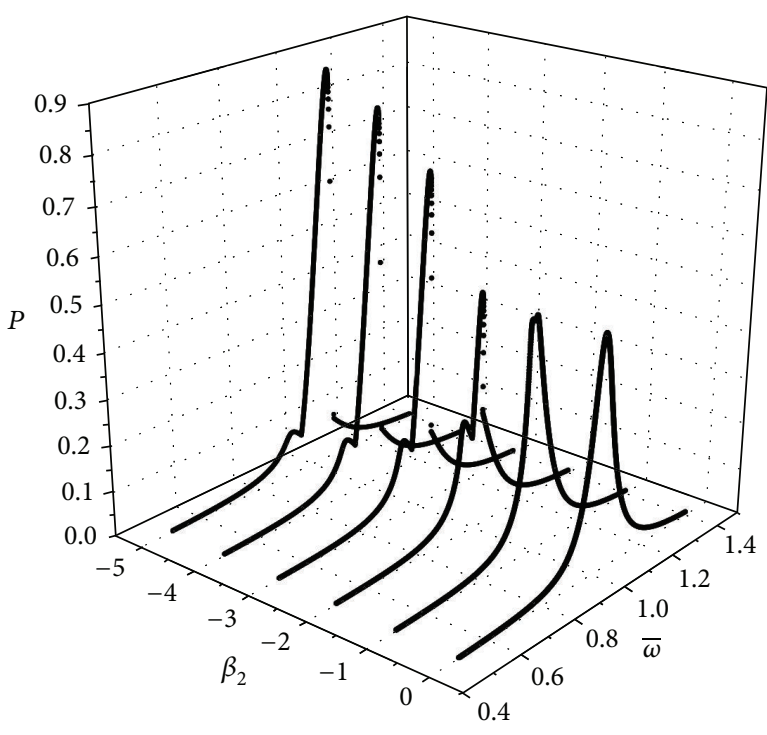

(a)

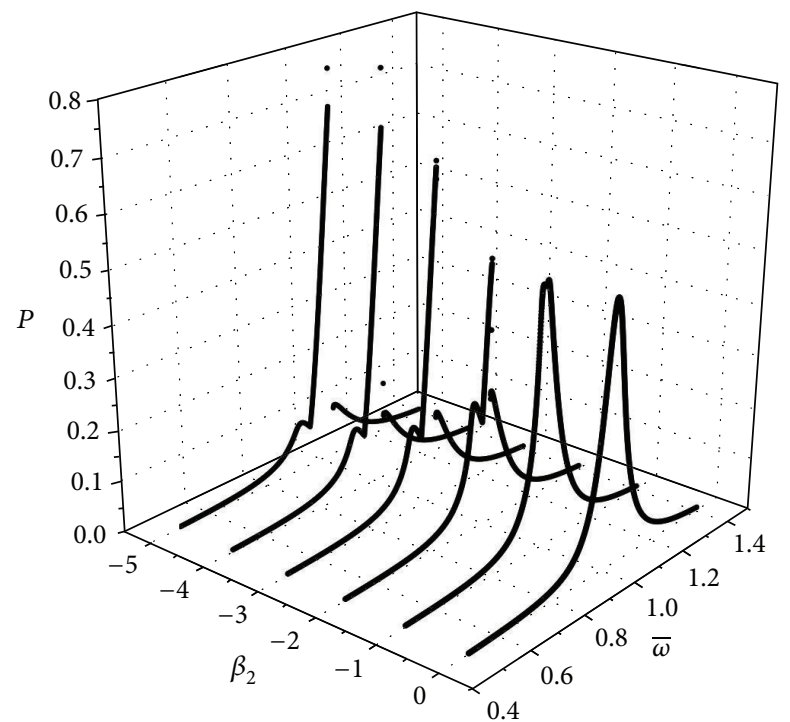

(b)

FIGURE 14: Maximum power amplitude versus frequency with $\rho=1$ for the LM and NLM-Q models; (a) upsweep and (b) downsweep.

is also changed in order to evaluate its influence on system dynamics.

Results are mainly presented as nondimensional displacement-frequency curves. Basically, maximum values of displacement are plotted under a slow quasi-static variation of the frequency. The last state at the previous forcing frequency is considered as the initial condition for the new frequency. Figure 12 shows the displacement-frequency curves for different models and three different nondimensional electrical resistive loads. Different values of nonlinear piezoelectric couplings $\left(\beta_{1}\right.$ and $\left.\beta_{2}\right)$ are analyzed. The left panel is related to the NLN-L while the right panel is associated with the
NLM-Q. The linear model is related to the situation where $\beta_{1}=0$ and $\beta_{2}=0$. Results show that nonlinear piezoelectric coupling variations change the peak frequencies and also increase system amplitude compared with linear model (LM), being an essential advantage for energy harvesting purposes. Note that the increase of the nonlinear piezoelectric couplings tends to deform the resonant curve and this effect is more pronounced with the increase of the resistance values. This deformation causes the appearance of dynamical jumps associated with discontinuities of the curves.

In order to present a better explanation related to dynamical jumps, the model NLM-Q is treated by considering high 


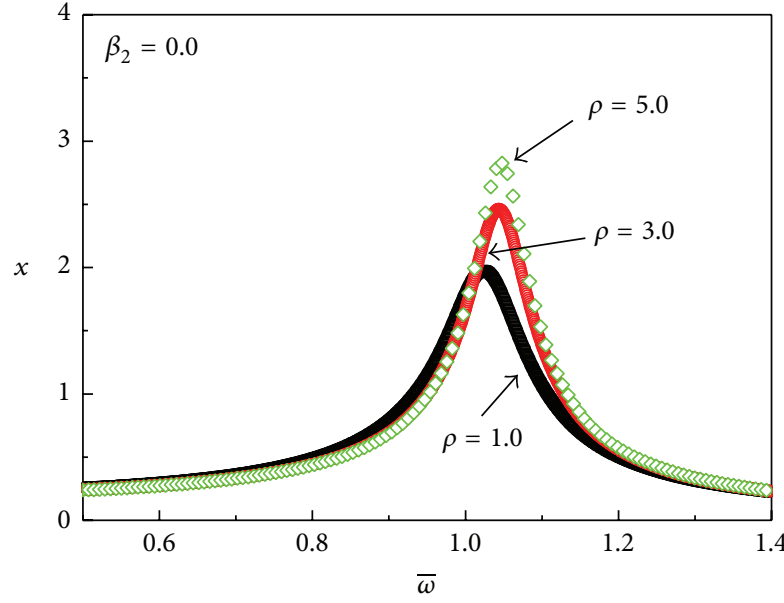

(a)

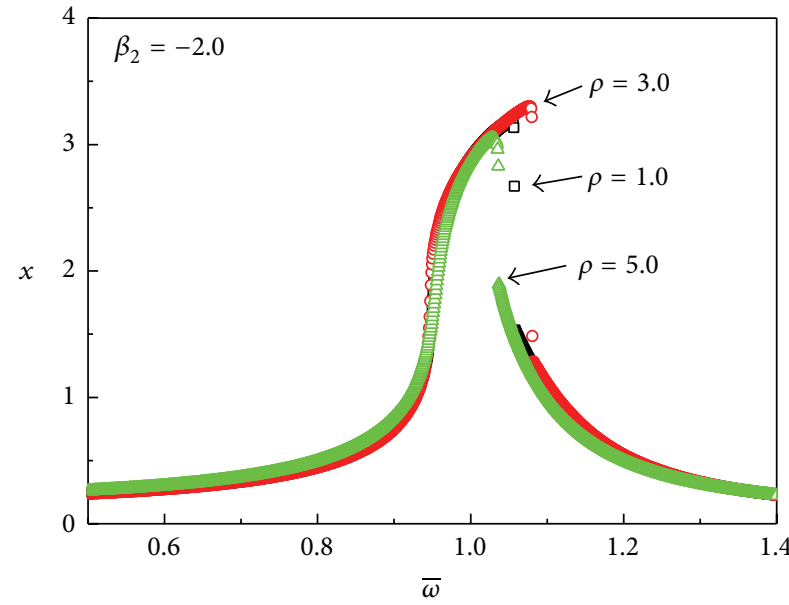

(b)

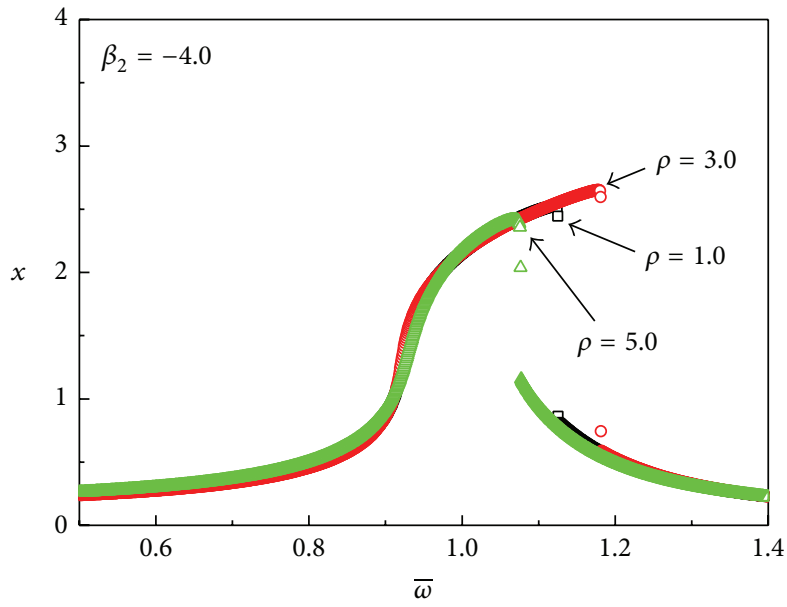

(c)

FIGURE 15: Maximum induced strain versus frequency for different values of $\rho$ for (a) $\beta_{2}=0.0$, (b) $\beta_{2}=-2.0$, and (c) $\beta_{2}=-4.0$ (upsweep).

values of $\beta_{2}$, with $\beta_{1}=0$ and $\rho=1$. Figure 13(a) shows the maximum system amplitude in steady state for frequency upsweep and Figure 13(b) presents the downsweep case. Note that the linear case is represented by $\beta_{2}=0$ and that the increase of $\beta_{2}$ causes the change of the resonant curve. Initially, the deformation of the curve is related to an increase of value but, afterward, this deformation is related to the side, promoting the appearance of dynamical jumps. Another important aspect is that the downsweep behavior is different from the upsweep. Figure 14 shows results of the maximum nondimensional power harvested as a function of frequency for similar situations (upsweep and downsweep). Note that the power increases near resonance and as expected, it is strongly influenced by dynamical jumps. These results are in close qualitative agreement with experimental data presented in the previous section.

The combination of piezoelectric coupling with electrical resistance can dramatically change system dynamics. Figure 15 shows the resonant curves obtained with upsweep test for different values of $\rho$, but also changing the nonlinear coupling effect. Figure 15(a) presents the linear model $\left(\beta_{1}=\beta_{2}=0\right)$, showing that the maximum amplitude is reached at different frequencies for different electrical loads. By increasing the modulus of $\beta_{2}$, it can be seen that the dynamical jumps occur by increasing frequency. The increase of nonlinear piezoelectric coupling is presented in Figures 15(b) and 15(c), showing the deformation of the curve that alters either the amplitude or the jumps.

Details of this kind of behavior are presented in Figure 16 for $\beta_{2}=-4.0$ and $\rho=5.0$, showing both upsweep and downsweep responses, highlighting the dynamical jumps. Figure 16(a) presents displacement curves, highlighting the phase space for situations before and after jumps. Figure 16(b) presents the power harvested for the same situations. These jumps are associated with dramatic changes that can reduce the response amplitude and the harvested energy.

\section{Conclusions}

This paper deals with the analysis of the nonlinear behavior influence of the piezoelectric element in vibration-based energy harvesting systems. Numerical simulations are carried 

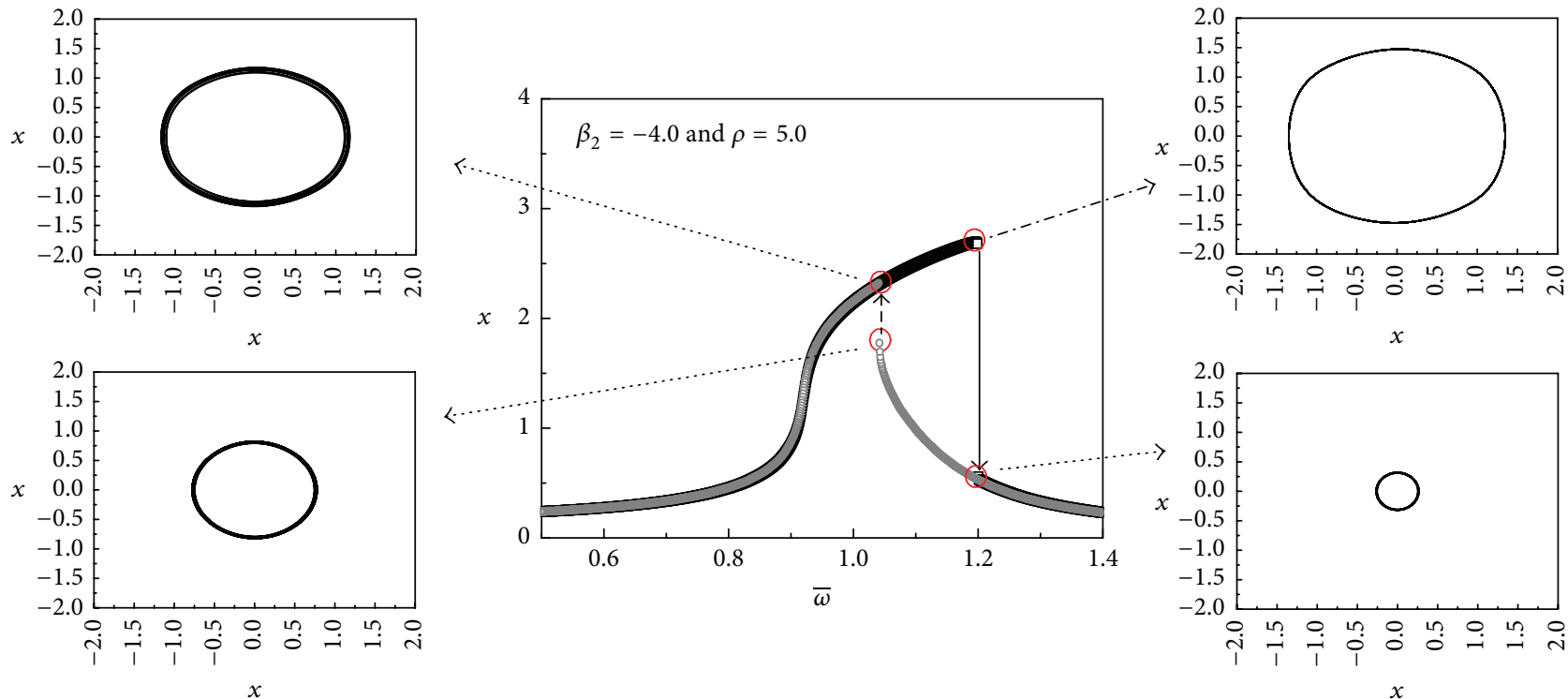

(a)
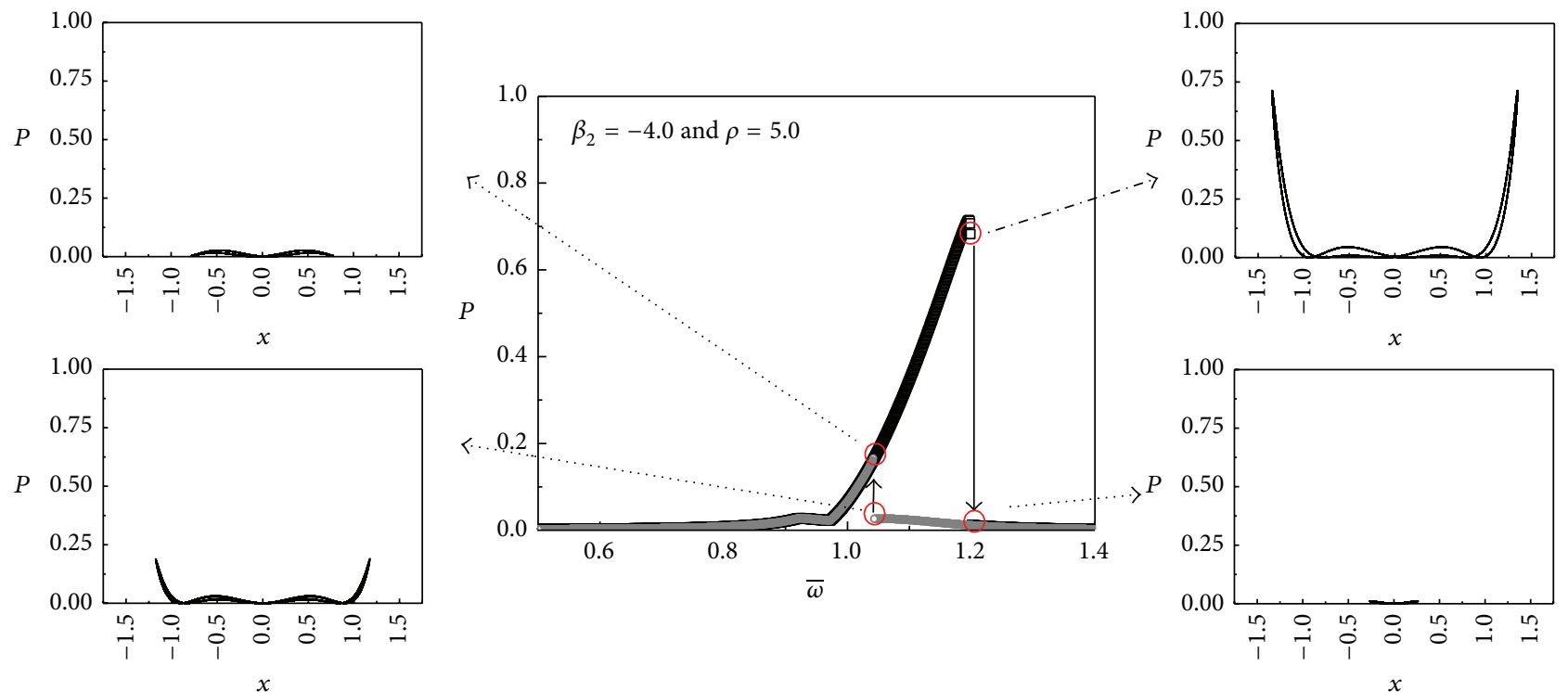

(b)

FIgURE 16: Dynamical jumps. (a) Resonant curves; (b) maximum nondimensional power harvested.

out considering linear and nonlinear models of piezoelectric coupling showing their influence on system dynamics. Three different models are treated for the piezoelectric coupling coefficient: linear model; nonlinear model with linear variation; and nonlinear model with quadratic variation. Experimental data from Kim et al. [23] are used as a reference. Results show that piezoelectric nonlinearity has a significant influence on the system performance in terms of the harvested power especially under resonant conditions. Quadratic nonlinear model captures the general behavior of the energy harvester, presenting good agreement with experimental data close to resonant conditions. Results suggest that the inclusion of nonlinear terms in the energy harvester models can be used to reduce discrepancies predicted by linear models. Although nonlinearities can enhance the power harvesting performance, it is important to observe that it can introduce complexity to system dynamics. Therefore, a deeper dynamical analysis is important for harvester design. Dynamical jumps are a possible critical situation associated with dramatic changes of system response.

\section{Conflict of Interests}

The authors declare that there is no conflict of interests regarding the publication of this paper. 


\section{Acknowledgments}

The authors would like to thank the Brazilian Research Agencies CNPq, CAPES, and FAPERJ through the INCTEIE (National Institute of Science and Technology, Smart Structures in Engineering) and FAPEMIG for their support. The Air Force Office of Scientific Research (AFOSR) is also acknowledged.

\section{References}

[1] H. A. Sodano, D. J. Inman, and G. Park, "A review of power harvesting from vibration using piezoelectric materials," The Shock and Vibration Digest, vol. 36, no. 3, pp. 197-205, 2004.

[2] A. Erturk and D. J. Inman, Piezoelectric Energy Harvesting, John Wiley \& Sons, Chichester, UK, 2011.

[3] A. S. De Paula, D. J. Inman, and M. A. Savi, "Energy harvesting in a nonlinear piezomagnetoelastic beam subjected to random excitation," Mechanical Systems and Signal Processing, vol. 54, pp. 405-416, 2015.

[4] A. Erturk, J. Hoffmann, and D. J. Inman, "A piezomagnetoelastic structure for broadband vibration energy harvesting," Applied Physics Letters, vol. 94, no. 25, Article ID 254102, 2009.

[5] A. Erturk, W. G. R. Vieira, C. De Marqui Jr., and D. J. Inman, “On the energy harvesting potential of piezoaeroelastic systems," Applied Physics Letters, vol. 96, no. 18, Article ID 184103, 2010.

[6] S. Roundy, D. Steingart, L. Frechette, P. Wright, and J. Rabaey, "Power sources for wireless sensor networks," in Wireless Sensor Networks, vol. 2920 of Lecture Notes in Computer Science, pp. 117, Springer, Berlin, Germany, 2004.

[7] N. E. Dutoit and B. L. Wardle, "Performance of microfabricated piezoelectric vibration energy harvesters," Integrated Ferroelectrics, vol. 83, no. 1, pp. 13-32, 2006.

[8] A. Triplett and D. D. Quinn, "The effect of non-linear piezoelectric coupling on vibration-based energy harvesting," Journal of Intelligent Material Systems and Structures, vol. 20, no. 16, pp. 1959-1967, 2009.

[9] P. D. Mitcheson, T. T. Toh, K. H. Wong, S. G. Burrow, and A. S. Holmes, "Tuning the resonant frequency and damping of an electromagnetic energy harvester using power electronics," IEEE Transactions on Circuits and Systems II: Express Briefs, vol. 58, no. 12, pp. 792-796, 2011.

[10] E. F. Crawley and E. H. Anderson, "Detailed models of piezoceramic actuation of beams," Journal of Intelligent Material Systems and Structures, vol. 1, no. 1, pp. 4-25, 1990.

[11] L. L. Silva, M. A. Savi, P. C. Monteiro, and T. A. Netto, "Effect of the piezoelectric hysteretic behavior on the vibration-based energy harvesting," Journal of Intelligent Material Systems and Structures, vol. 24, no. 10, pp. 1278-1285, 2013.

[12] G. K. Ottman, H. F. Hofmann, and G. A. Lesieutre, "Optimized piezoelectric energy harvesting circuit using step-down converter in discontinuous conduction mode," IEEE Transactions on Power Electronics, vol. 18, no. 2, pp. 696-703, 2003.

[13] E. Lefeuvre, A. Badel, C. Richard, L. Petit, and D. Guyomar, "A comparison between several vibration-powered piezoelectric generators for standalone systems," Sensors and Actuators A: Physical, vol. 126, no. 2, pp. 405-416, 2006.

[14] A. Badel, D. Guyomar, E. Lefeuvre, and C. Richard, "Efficiency enhancement of a piezoelectric energy harvesting device in pulsed operation by synchronous charge inversion," Journal of
Intelligent Material Systems and Structures, vol. 16, no. 10, pp. 889-901, 2005.

[15] E. Lefeuvre, A. Badel, C. Richard, and D. Guyomar, "Piezoelectric energy harvesting device optimization by synchronous electric charge extraction," Journal of Intelligent Material Systems and Structures, vol. 16, no. 10, pp. 865-876, 2005.

[16] M. Lallart, C. Magnet, C. Richard et al., "New synchronized switch damping methods using dual transformations," Sensors and Actuators A: Physical, vol. 143, no. 2, pp. 302-314, 2008.

[17] H. Shen, J. Qiu, H. Ji, K. Zhu, and M. Balsi, "Enhanced synchronized switch harvesting: a new energy harvesting scheme for efficient energy extraction," Smart Materials and Structures, vol. 19, no. 11, Article ID 115017, 2010.

[18] S. C. Stanton, A. Erturk, B. P. Mann, and D. J. Inman, "Nonlinear piezoelectricity in electroelastic energy harvesters: modeling and experimental identification," Journal of Applied Physics, vol. 108, no. 7, Article ID 074903, 2010.

[19] M. F. Daqaq, "Response of uni-modal duffing-type harvesters to random forced excitations," Journal of Sound and Vibration, vol. 329, no. 18, pp. 3621-3631, 2010.

[20] A. Erturk and D. J. Inman, "Broadband piezoelectric power generation on high-energy orbits of the bistable Duffing oscillator with electromechanical coupling," Journal of Sound and Vibration, vol. 330, no. 10, pp. 2339-2353, 2011.

[21] M. A. Karami and D. J. Inman, "Equivalent damping and frequency change for linear and nonlinear hybrid vibrational energy harvesting systems," Journal of Sound and Vibration, vol. 330, no. 23, pp. 5583-5597, 2011.

[22] W. Martens, U. von Wagner, and G. Litak, "Stationary response of nonlinear magneto-piezoelectric energy harvester systems under stochastic excitation," European Physical Journal: Special Topics, vol. 222, no. 7, pp. 1665-1673, 2013.

[23] M. Kim, M. Hoegen, J. Dugundji, and B. L. Wardle, "Modeling and experimental verification of proof mass effects on vibration energy harvester performance," Smart Materials and Structures, vol. 19, no. 4, Article ID 045023, 2010. 

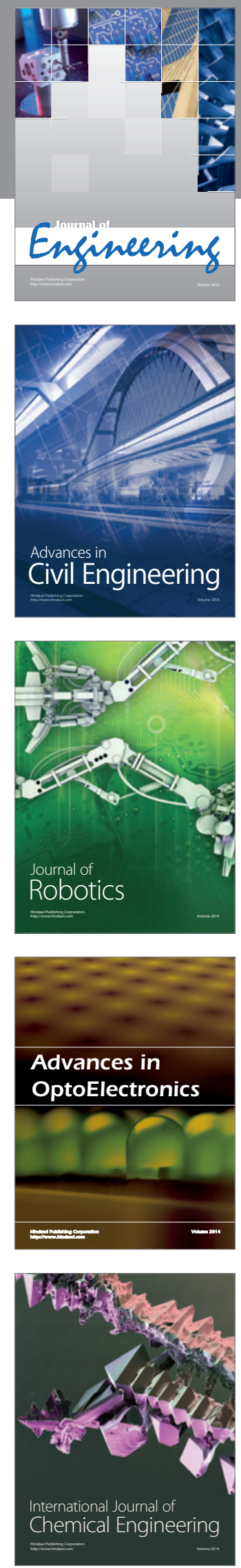

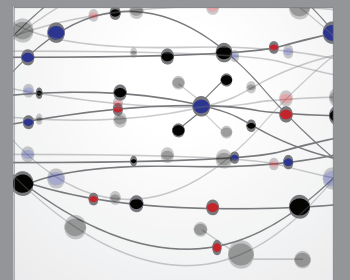

The Scientific World Journal
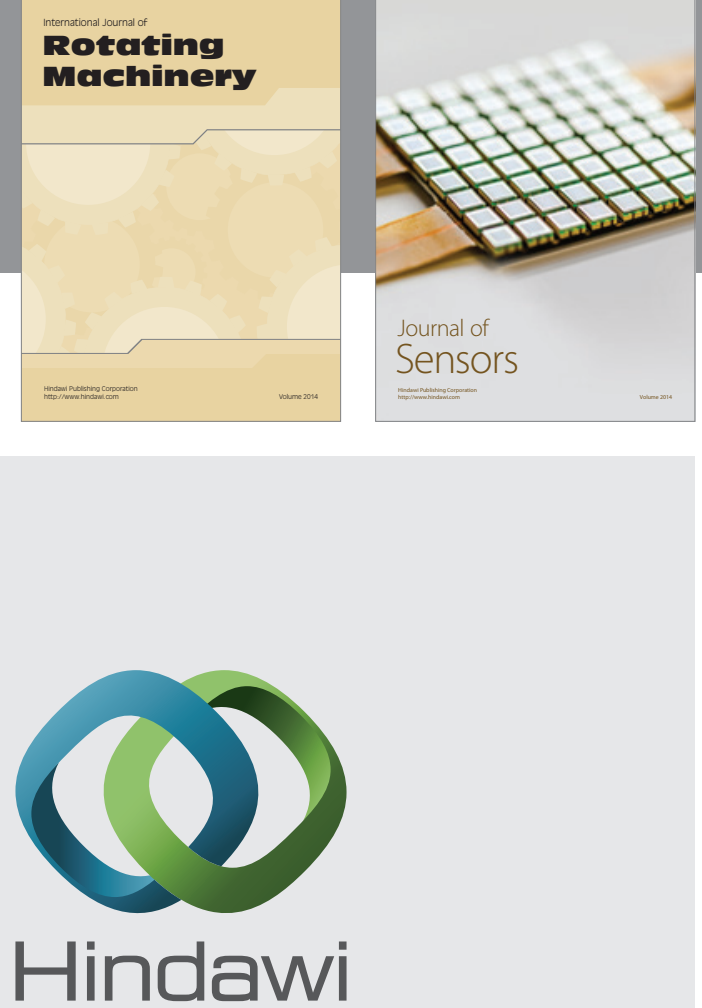

Submit your manuscripts at http://www.hindawi.com
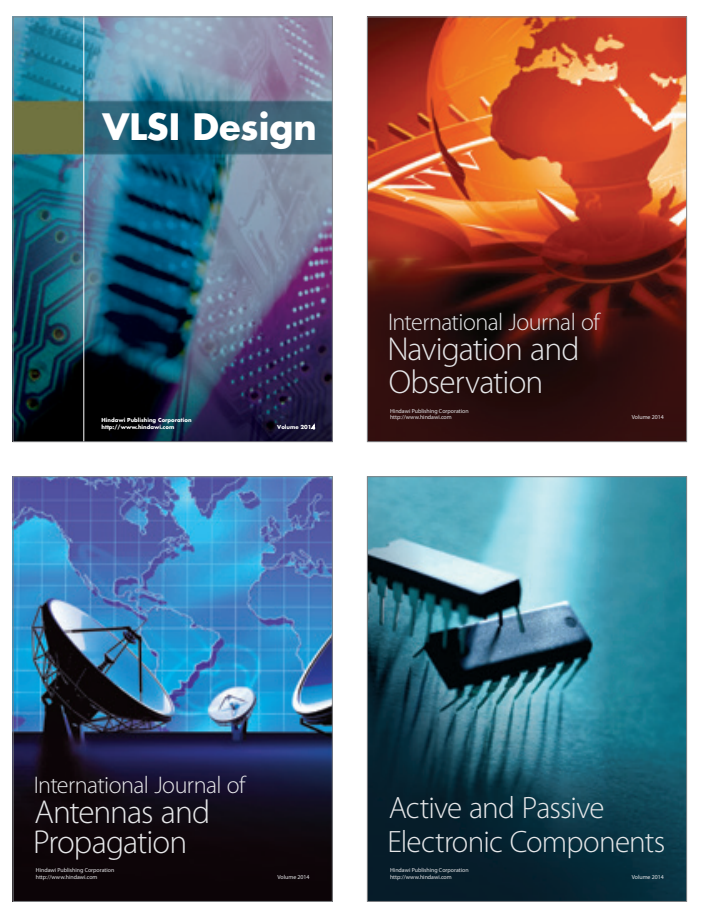
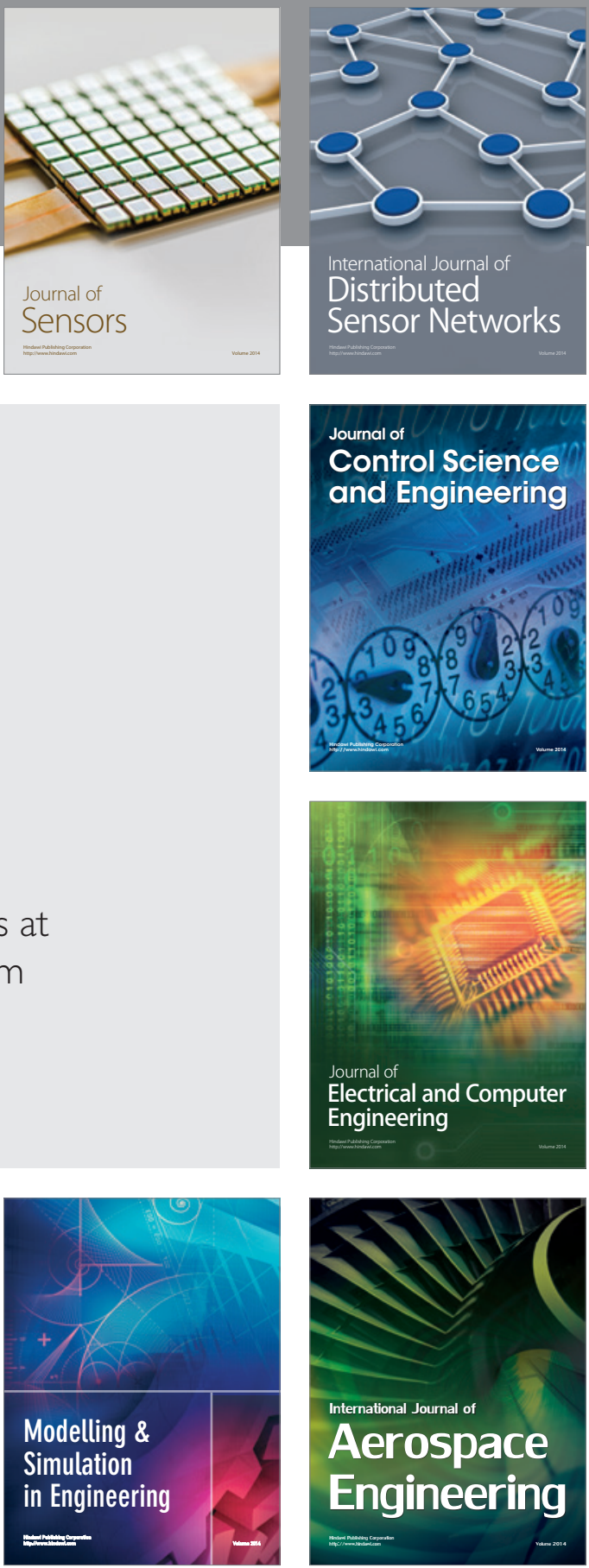

Journal of

Control Science

and Engineering
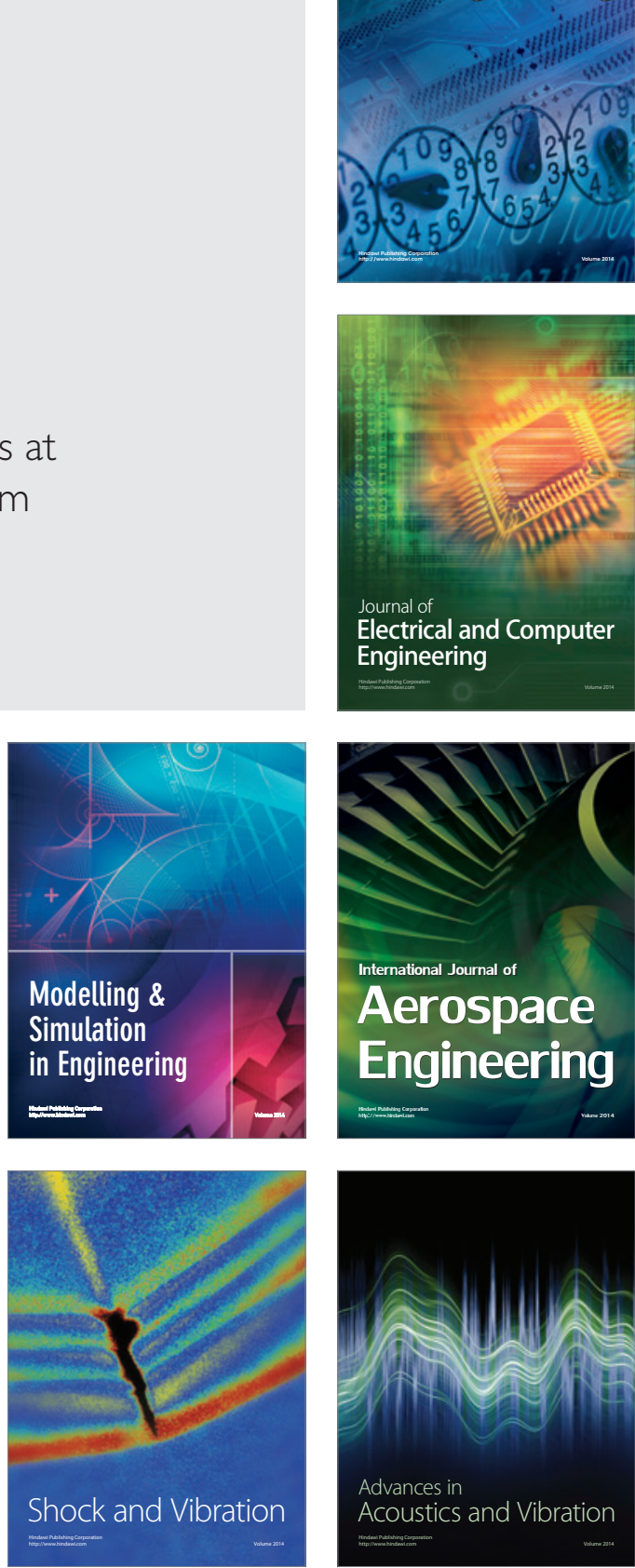